\title{
Daphnia carinata genome provides insights into reproductive switching
}

\author{
Jingyi Jia ${ }^{1}$, Guangfu Hu${ }^{1}$, Cui Feng ${ }^{1}$, Chenchen Dong ${ }^{1}$, Mengqi Han ${ }^{1}$, and Xiangjiang Liu ${ }^{2}$ \\ ${ }^{1}$ Huazhong Agricultural University \\ ${ }^{2}$ Affiliation not available
}

June 2, 2020

\begin{abstract}
The water flea Daphnia carinata is emerging as a new model for biological studies due to its natural cyclical parthenogenesis and small genome. However, no genomic information for the D. carinata is currently available. Here, we reported a chromosomescale genome assembly of D. carinata. The assembled D. carinata (WSL) genome was consisted of $131.58 \mathrm{Mb}$, and $92.23 \%$ $(121.36 \mathrm{Mb})$ of the assembly was anchored onto 10 chromosomes. Basing on the whole genome information, we further compared the transcriptomic and epigenomic characterization among parthenogenetic females (PF), sexual females (SF) and males (M) in D. carinata. Transcriptomic analysis showed that the highly expressed genes in M were mainly grouped into the cuticle, phototransduction, hypoxia-response, sex differentiation, and methyl farnesoate synthesis. Besides, compared to M and SF, the highly expressed genes in PF were mainly grouped into energy metabolism, mitosis, DNA replication, and RNA splicing. miRNAomic analysis showed that several miRNA-mRNA pairs may be the functional modules in the reproductive regulation of D. carinata, such as novel-miR-4 - brat, novel-miR-21-lin-9 pairs, novel-miR-110 - gl, novel-miR-4 - eIF3-S9. The wholegenome DNA methylation analysis showed that genome-wide methylation rate was very low in D. carinata, and DNA methylation sites were mainly enriched in the gene body region. Comparative analysis showed that the genome-wide methylation rate in $\mathrm{PF}$ was higher than that in SF and M. Differentially methylation region-related genes were all mainly grouped in metabolic process, catalytic activity, binding/ion binding, and cellular process.
\end{abstract}

\section{Introduction}

The water flea Daphnia carinata(Cladocera, Daphniidae, $2 \mathrm{~N}=20$ ) is a widespread freshwater microcrustacean (Zhang, Zeng, Chen, \& Zhao, 2009), which is emerging as a new model for ecology (Jiang et al., 2014), phylogeny (Geng, Cheng, Deng, \& Zhang, 2016), toxicology (Lekamge, Miranda, Ball, Shukla, \& Nugegoda, 2019), and physiology studies (Wiggins \& Frappell, 2000). D. pulex and D. magna, as the established models, have a fairly complete genomic resource. D. pulex has completed whole-genome sequencing for two different strains, including TCO strain (Colbourne et al., 2011) and PA42 strain (Ye et al., 2017), respectively. Similarly, the whole genome sequences of D. magna have also been completed in two different strains, including Xinb3 strain (Colbourne, Singan, \& Gilbert, 2005) and SK strain(Lee et al., 2019), respectively. However, to date, no genomic information is available for $D$. carinata, which greatly limits the promotion of $D$. carinata as a model.

Similar to other cladocerans, $D$. carinata could also display the cyclical parthenogenesis, that is, switching from parthenogenetic (asexual) to sexual reproduction when the living environmental characteristics deteriorate (Dinh et al., 2018; Kong et al., 2016). For reproductive switching, previous studies indicated that the occurrence of male Daphnia could be induced by juvenile hormone analogs (Olmstead \& LeBlanc, 2003; 
Tatarazako, Oda, Watanabe, Morita, \& Iguchi, 2003). In addition, several reproductive switching-related genes have also been detected in daphnia, such as doublesex1, transformer ,dsx /mab-3 domain, VTN , $V T G$ and HSPgenes (Kato et al., 2008; Kato et al., 2010; Kato, Kobayashi, Watanabe, \& Iguchi, 2011; Xu et al., 2009). Whereas, little is known about the global comparation among parthenogenetic females (PF), sexual females (SF) and males (M) in daphnia by using high-throughput sequencing technology.

DNA methylation, as a form of epigenetic control mechanism, plays a major role in the regulation of gene expression (Genetics \& Maher, 2011). Until now, epigenetic studies in Daphnia were mainly focused on environment toxicology (Athanasio, Sommer, Viant, Chipman, \& Mirbahai, 2018; Kim, Koedrith, \& Seo, 2015), evolutionary biology (Asselman, De Coninck, Pfrender, \& De Schamphelaere, 2016; Kvist et al., 2018), and genetics (Hearn, Pearson, Blaxter, Wilson, \& Little, 2019; Strepetkaite et al., 2016). While little knowns about the functional role of DNA methylation on reproductive biology in Daphnia . Interestingly, our recent study found that the methyltransferase gene products were significantly different between PF and SF in Moina micrura(Cladocera, Moinidae, Moina) (Jia, J. Y. et al., 2018). These studies suggested that DNA methylation might play a key role in daphnia reproductive switching.

In this study, firstly, the chromosome-level genome of $D$. carinata was constructed in order to facilitate further studies for this model. Then, basing on the genome data, the transcriptional (mRNA and miRNA) genetic characteristics were compared among $\mathrm{PF}, \mathrm{M}$, and $\mathrm{SF}$ in $D$. carinata. Finally, the whole-genome DNA methylations were also compared among PF, M, and SF in D. carinata. These studies will help us to acquire novel molecular features that may underlie aspects of the $D$. carinata adaptation to environmental stress, and provide a foundation for further clear the molecular basis of reproductive switching in Daphnia .

\section{Materials and Methods}

\section{Animals}

The clone of $D$. carinata (WSL strain) was originally isolated from the South Lake (Wuhan, Hubei, China), and the pure lines were maintained in our laboratory. Parthenogenetic female individuals were harvested for genomic DNA sequencing and Hi-C library construction. To cover a comprehensive molecular feature that individual-specifically expressed in DNA methylation, mRNA and microRNA level, parthenogenetic female, sexual female and male D. carinata were identified and collected, respectively (Figure 1). All samples were snap-frozen in liquid nitrogen and then stored at $-80^{\circ} \mathrm{C}$ until further processing.

\section{Genome sequencing}

Genomic DNA was used to construct both Illumina 270-bp insert library (123.49X) and PacBio 20-kb insert library (80X), and sequenced on Illumina HiSeq 2500 and PacBio SMRT platforms, respectively. To construct the chromosome-scale genome, the high-throughput chromosome conformation capture (Hi-C) library was prepared and then sequenced on Illumina HiSeq X 10 platform (Supplementary Methods).

\section{Genome survey}

To facilitate the development of a customized sequencing and assembly strategy, the 19-Kmer frequency distribution was used to estimate the genome size, repeat sequence ratio, and heterozygosity. The estimated $D$. carinata genome size was to be $\sim 128.60 \mathrm{Mb}$, the estimated repeated sequence ratio was about $31.28 \%$ and the estimated heterozygosity was about $0.42 \%$ (Supplemental Figure S1). Thus, the genome survey suggested that the sequencing strategy by using Illumina and PacBio data was suitable for $D$. carinata genome (Supplementary Methods). 


\section{Genome assembly}

The clean PacBio reads were corrected by using Canu software with the default parameters. Subsequently, wtdbg (https://github.com/ruanjue/wtdbg) was chosen to perform the initial contigs assembly of the $D$. carinata, and the assembly results were corrected by Illumina data using Pilon. Finally, Illumina sequencing reads alignment rate, CEGMA analysis and BUSCO analysis were used to evaluate the accuracy and completeness of the assembly (Simao, Waterhouse, Ioannidis, Kriventseva, \& Zdobnov, 2015). The chromosomallevel genome was assembled using LACHESIS software with Hi-C library (Burton et al., 2013; Imakaev et al., 2012) (Supplementary Methods).

\section{Genome annotation}

The $D$. carinata genome repetitive elements library was constructed according to the principle of structural prediction and de novoprediction. The $D$. carinata genome repetitive elements library was constructed basing on the principle of structural prediction and de novo prediction. The repetitive elements were classified using PASTEClassifier (Seberg \& Petersen, 2009) and combined with Repbase database (Bao, Kojima, \& Kohany, 2015) to create the final repeat library. And then, we used RepeatMasker program v4.0.6 (Chen) to predict repetitive elements in the $D$. carinata genome based on the constructed repeat sequence database. Gene prediction was carried out using de novo prediction, homology-based method, and RNAseq-based approach. Then, the evidence, from the three above independent predictions, was integrated using EVM v1.1.1 (Haas et al., 2008). Annotation of the predicted genes was performed by aligning the gene sequences to the NR, KOG, KEGG, and TrEMBL databases with BLAST v2.2.31. Basing on the Rfam database and the miRBase database, ribosomal and microRNA were respectively predicted using Infenal v1.1 (Nawrocki \& Eddy, 2013). And transfer RNA genes were predicted by using tRNAScan-SE v1.3.1 algorithm (Lowe \& Eddy, 1997). Finally, pseudogenes were identified with GenBlastA v1.0.4 and GeneWise v2.4.1 (Supplementary Methods).

\section{mRNA and miRNA expression profiling}

RNA-seq reads and miRNA-seq reads were generated by Illumina HiSeq 2000 system. The high-quality mRNA reads were mapped to the D. carinata genome using Tophat (v2.0.9) (Trapnell, Pachter, \& Salzberg, 2009). The gene expression was measured by the fragments per kilobase of exon per million mapped reads (FPKM) value. Differentially expressed genes (DEGs) were determined by edgeR. GO term and KEGG pathway enrichment analysis were respectively performed by goatools and KOBAS, and only significantly enriched GO terms and KEGG pathways were reported (Bonferroni-corrected P-value[?]0.05). Subsequently, the hub genes were identified by the MCODE plugin and the CytoHubba plugin, and were visualized by Cytoscape (Version 3.7.1) (Shannon et al., 2003). The cleaned sRNAs reads were aligned to the D. carinata genome sequences via Bowtie (Langmead, Trapnell, Pop, \& Salzberg, 2009). Conserved miRNAs were annotated with miRbase 22, and novel miRNAs were identified using the miRDeep2 (Friedlander, Mackowiak, Li, Chen, \& Rajewsky, 2012). The miRNA expression was measured by transcripts per kilobase million (TPM) value. Differentially expressed miRNAs (DEMs) were identified by edgeR. The potential targets for known and novel miRNAs were predicted by miRanda software (Enright et al., 2004), and the expression levels of their target genes were identified in our RNA-seq analysis. The differential expression patterns of the genes or miRNAs were further validated by qRT-PCR analysis (Table S1; Supplementary Methods). 


\section{Integration analysis of transcription factor (TF), mRNA and miRNA expression profiles}

DEMs and DEGs were extracted according to the results of the differential expression analysis, and then constructed the TF - miRNA - mRNA regulatory network using a combination of TF-binding site analysis and miRNA target gene data (Supplementary Methods).

\section{BS-seq library construction and Quality control}

A total amount of $5.2 \mu \mathrm{g}$ genomic DNA spiked with $26 \mathrm{ng}$ lambda DNA was fragmented to 200-300 bp with a Covaris S220 sonicator, followed by end repair and adenylation. Then, these DNA fragments were treated twice with bisulphite using the EZ DNA Methylation-Gold Kit. BS-seq libraries were sequenced on the Illumina Hiseq 2500 platform. FastQC (fastqc_v0.11.5) was utilized to perform basic statistics on the quality of the raw reads. Then, those reads sequences generated by the Illumina pipeline were pre-processed through Trimmomatic software. Finally, the clean reads were obtained and used for all subsequent analyses (Supplementary Methods).

\section{Reads mapping and compare analysis}

Bismark software was used to perform alignments of bisulphite-treated reads to the $D$. carinata genome using default parameters (Krueger \& Andrews, 2011) (with an average of $52 \%$ Unique Mapping rate). 10kb slidingwindow approach was applied for methylation-level analysis. The methylation level (\%) was computed by dividing the number of reads of each methylated cytosine $(\mathrm{mC})$ by the total reads for that cytosine $(\mathrm{C})$, as follows: $\mathrm{ML}(\%)=$ reads $(\mathrm{mC}) /($ reads $(\mathrm{mC})+$ reads $(\mathrm{C})) \mathrm{X} 100$, a significance threshold of q-value[?]0.01 was applied. CpG sites were the predominant DNA methylation sites in invertebrate (Song, Li, \& Zhang, 2017; Wang et al., 2013; Xiang et al., 2010) and have demonstrated mostly DNA methylation occurs at CpG sites in crustaceans D. pulex and D. magna (Asselman et al., 2016; Hearn et al., 2019). Therefore, statistical analyses of DNA methylation in D. carinata were mainly performed at the $\mathrm{CpG}$ site level. Differentially methylated regions (DMRs) contained at least three CG sites with $\mathrm{p}<1 \mathrm{e}-05$ (Wald test) in methylation levels were identified by DSS software (Feng, Conneely, \& Wu, 2014; Park \& Wu, 2016; Wu et al., 2015). After DMRs were identified, genes located in DMRs were characterized. The DMR-associated genes and promoters were analyzed for GO and KEGG enrichment. GO terms or pathways with Bonferroni-corrected $P$ -value less than 0.05 were considered significantly enriched by DMR-related genes or promoters. Statistical analyses of DNA methylation for M, PF and SF were performed at two different levels, viz. the CpG site level and the region level (Supplementary Methods).

\section{Results}

\section{Genome sequencing, assembly, and characterization}

The complementary approaches were brought together to develop D. carinata reference genome assembly (Figure 2 and Table S2). Firstly, basing on K-mer statistics, the genome size of D. carinata was estimated to be ${ }^{\sim} 128.60 \mathrm{Mb}$ (Figure S1 and Table S3). Then, the final assembled genome size was $131.59 \mathrm{Mb}$ with a contig N50 size of $2.80 \mathrm{Mb}$ and a scaffold N50 size of $8.41 \mathrm{Mb}$, and the longest scaffold spanned 14.95 $\mathrm{Mb}$ (Table 1). Finally, Hi-C linking information was further anchored onto 10 chromosomes (Figure 2 and Figure S2) with more than $92.23 \%$ of contig sequences were located on the chromosomes (Table S4). 
The assembly quality and completeness analysis demonstrated that $89.13 \%$ Illumina sequencing reads were mapped to the assembly of the genome, 457 (99.78\%) cluster of essential genes (CEGs) were hit in the assembled genome, and $96.5 \%$ of conserved core terrestrial animal genes were completely detected in the assembled genome (279: complete and single-copy, 17: complete and duplicated) among 303 tested BUSCOs (Tables S5a, b\&c). A set of 21,982 protein-coding genes were predicted based on the integration of evidence from de novo prediction, homology data, and transcriptome (Tables S6\&7 and Figure S3), of which 87.03\% $(19,132)$ were annotated based on known proteins in public databases (Table S8). Specifically, 9,709 $(44.17 \%)$, $7423(33.77 \%), 11,563(52.60 \%), 18,945(86.18 \%)$, and 18,978 (86.33\%) reference genes were respectively annotated with the GO, KEGG, NR, KOG, and TrEMBL databases (Table S8). The repetitive elements accounted for $23.51 \%$ (30.94 $\mathrm{Mb}$ ) of the reference genome (Table S9), dominated by transposable elements (16.77\%). Additionally, 4,399 tRNAs, 132 rRNAs, 18 microRNAs, and 998 pseudogenes were identified in the $D$. carinata genome (Table S10).

\section{Transcriptomic analysis among SF, PF and $\mathrm{M}$ inD. carinata}

To identify genes associated with reproductive switching, the cDNA libraries were constructed for the SF, $\mathrm{PF}$, and $\mathrm{M}$, and $88.90 \%^{\sim} 88.95 \%$ clean reads were matched to D. carinata genomic locations (Table S11). The DEGs (differential expressed genes) among SF, PF, and M were analyzed (Supplementary Information), and the result indicated that most of DEGs were mainly distributed in chromosome 1 and chromosome 5 (Figures S4A, B\&C). Moreover, we constructed PPI networks and selected hub genes using cytoHubba and MCODE (Supplementary Methods, Tables S12-17 and Figures S5-7). Besides, qRT-PCR results indicated that randomly selected 24 DEGs were differentially expressed and the expression pattern was matched with NGS data analysis (Table S18).

\section{Key GO terms and pathways involved in the reproductive switching in D. carinata}

GO enrichment analysis demonstrated that the upregulated genes in SF compared to PF were mainly enriched in cuticle, hemoglobin, stress, and methylation (Figure 3, SF vs PF); similarly, the mostly enriched terms for upregulated genes in SF compared to M were hemoglobin, reproduction, methylation, and glutamate receptor signaling pathway (Figure 3, M vs SF). Secondly, the upregulated genes in M compared to PF were mainly enriched in phototransduction, cuticle, receptor activity, and hormone activity (Figure 3, M vs PF); and the most frequently enriched terms in M compared to SF included phototransduction, cuticle, signal transduction, and hormone activity (Figure 3, M vs SF). Finally, upregulated genes in PF compared to SF were mainly enriched in lipid transport, ionotropic glutamate receptor signaling pathway, DNA packaging, transcription, and transferase activity (Figure 3, SF vs PF); similarly, the most frequently enriched terms in $\mathrm{PF}$ compared to M included DNA packaging, transcription, and lipid transport (Figure 3, M vs PF).

Basing on the pathway enrichment analysis, several noteworthy pathways were further dug out (Figure 4 and Table 2). Firstly, main stress signaling pathway (PI3K-Akt and MAPK pathways) was found to be upregulated in M compared to PF or SF. Besides, compared to PF and M, steroid biosynthesis pathway was upregulated in SF.

\section{DEGs related to reproductive switching}

Based on the analysis of hub genes and GO/pathway enrichment, we identified 59 upregulated and 40 downregulated reproductive switching-related genes in SF compare to PF (Table S19\&20), such as upregulated cuticle genes, reproduction-related genes, oxygen transport-related genes, stress classes and down- 
regulated hormone synthesis-ralated genes, vitellogenin-family genes; 57 upregulated and 68 downregulated reproductive switching-related genes in M compare to PF (Table S21\&22), such as upregulated cuticle genes, eproduction-ralated genes, phototransduction genes, oxygen transport-related genes and downregulated vitellogenin-family genes, splicing-related geness; 51 upregulated and 65 downregulated reproductiverelated genes in M compare to SF (Table S23\&24), such as upregulated cuticle genes, sex differentiation gene, phototransduction genes, signal transduction genes and downregulated reproduction-ralated genes, methylation genes, stress classes.

miRNAomic analysis among PF, SF and M to examine the key miRNAs involved in reproductive switching in D. carinata

The overall analysis of miRNAomic data showed that 34 conserved miRNAs (Supplementary Table S25) and 121 novel miRNA candidates (Supplementary Table S26) were identified in D. carinata . Furthermore, the DEMs among SF, PF, and M were analyzed (Tables S27-29; Supplemental Information), and qRT-PCR results indicated that randomly selected 3 DEMs were differentially expressed and the expression pattern was matched with NGS data analysis (Table S18).

Subsequently, according to the transcriptome-wide combined analysis of DEMs and DEGs, several miRNAmRNA pairs and upstream transcription factors (TFs) of miRNA were both identified (Figures 5-7). Interaction analysis showed that 26 DEMs targeted 103 DEGs, 43 TFs belonged to HB-other, C2H2, LIM, C2C2-GATA, and bZIP family. Here, 127 miRNA-mRNA pairs displayed negatively correlated expression profiles. In these negative pairs, several miRNA-mRNA regulatory modes might play key roles in the regulation of reproductive switching to response environment stress in D. carinata, such as novel-miR-110-gl , novel-miR-21-lin-9 , and novel-miR-4-eIF3-S9 pairs in M vs. PF (Figure 6, M vs. PF); novel-miR-4brat, novel-miR-21-lin-9 pairs in M vs. SF (Figure 7, M vs. SF). Finally, we found that some upstream transcription regulators of miRNA were involved in the compound eye development (such as Kr, B-H2, caup, exd, hth, Optix and so) (Figure 5-7).

\section{Genome-wide methylation analysis among SF, PF and M to identify the methylation markers involved in reproductive switching in $D$. carinata}

To explore the functional role of DNA methylation in the reproductive switching, the genome-wide methylation analysis was performed in three reproductive status (SF, PF, and $\mathrm{M})$ in D. carinata. The results showed that the $D$. carinata displayed a low methylation (1.17\%). To characterize the distribution of methylation in the different genomic regions, we further analyzed the global DNA methylation difference in six different gene subregions (promoter, 5'UTR, exon, intron, 3’UTR, repeat) (Figure 8B) and the upstream \& downstream of gene (TSS2000, gene body, TES2000) (Figure 8C). The results displayed that the distribution patterns of most methylated sites at the different genome functional elements were similar among SF, PF and M. The DNA methylation sites were mainly enriched in the gene body followed by the promoter, TSS2000, TES2000, exon and repeat (Figures 8B\&C). Furthermore, compared to PF, all genomic regions exhibited higher methylation levels in $\mathrm{SF}$ and $\mathrm{M}$ (Figures $8 \mathrm{~B} \& \mathrm{C}$ ), while the genomic exon region exhibited a higher methylation level in SF compared to M (Figure 8B).

Compare analysis showed that there were small methylation differences among SF (1.17\%), PF (1.19\%), and M $(1.16 \%)$ in D. carinata (Figure 8A). Subsequently, the differentially methylated regions (DMRs) were also compared among SF, PF and M. The results showed that more DMRs were identified between M and SF (552 hyper-DMRs and 552 hypo-DMRs; Figure 9A). Additionally, the hypomethylation was found in high transposon element coverage region (Figure 9A). Compared to hyper DMR-related genes and promotors, more hypo DMR-related genes and promoters were identified between M vs. SF, while the opposite pattern was detected in SF vs. PF and M vs. PF (Figure 9B). Besides, more DMR-related genes and promotors were detected in M vs. SF (Figure 9B). To investigate the regulatory role of differential methylation region-related genes or promotors, GO enrichment analyses indicated that DMR-related genes or promotors were mainly 
grouped in metabolic process, catalytic activity, binding/ion binding, and cellular process (Figure 10).

To further understand the regulatory mechanism of DNA methylation, the relationships between differentially methylated genes (DMGs) and DEGs were explored (Table S30). The results displayed that most of DMGs were not overlapped with DEGs, only $66(6.82 \%)$ genes displayed positive or negative correlation between methylation and mRNA expression level (Table S30), and positive and negative correlation were both presented in gene body or promotor regions. These results indicated that the functional relevance between DMGs and DEGs was confusing inDaphnia. Interestingly,vitelline membrane outer layer protein I-like protein, a noteworthy DMG (promotor region), was more abundantly detected in SF compared to M or PF.

\section{Dicussion}

To our knowledge, the first chromosome-scale genome ofDaphnia was constructed based on Hi-C assembly in our study. We observed that the size of genome assembly $(131.59 \mathrm{Mb})$ in $D$. carinata WSL strain was between the other two species of the genusDaphnia, viz. D. magna (122.94 129.54 Mb) (Colbourne et al., 2005; Lee et al., 2019) and D. pulex(156.42 $197.21 \mathrm{Mb}$ ) (Colbourne et al., 2011; Ye et al., 2017), and the assembly with a scaffold N50 size of $8.41 \mathrm{Mb}$, which is similar to the recently assembled $D$. magna SK strain with a long scaffold compared with other assembled species of genusDaphnia(Lee et al. 2019) (Supplemental Table S31). The CEGMA and BUSCO values of our assembly were $99.78 \%$ and $96.5 \%$, respectively, and $89.13 \%$ illumina sequencing reads mapping to the assembly of our genome, which suggests the genome of the D. carinata assembled is of high quality. Based on K-mer analysis, the D. carinata genome is low heterozygosity, similar to D. magna (Lee et al., 2019).

\section{Transcriptomics of reproductive switching in D. carinata}

Profiling of DEGs during reproductive switching, and subsequent functional and pathway analysis provided a comprehensive view of response to environmental stresses during the reproductive switching in D.carinata

In GO enrichment analysis, we found that the upregulated genes in SF compared to PF were mainly enriched in cuticle, hemoglobin, stress, and methylation; similarly, the mostly enriched terms for upregulated genes in SF compared to $\mathrm{M}$ were hemoglobin, reproduction, methylation, and glutamate receptor signaling pathway. Those upregulated genes in SF might play an important role in producing a protective cuticle structure to resist unfavorable conditions (Pesch, Riedel, Patil, Loch, \& Behr, 2016), increasing oxygen transport and storage to survive in hypoxic (low oxygen) water conditions (Pirow, Baumer, \& Paul, 2001), and contributing to the maturation of the gonads for producing haploid eggs during sexual reproductive period. Secondly, the upregulated genes in $\mathrm{M}$ compared to $\mathrm{PF}$ were mainly enriched in phototransduction, cuticle, receptor activity, and hormone activity; and the most frequently enriched terms in M compared to SF included phototransduction, cuticle, signal transduction, and hormone activity. Those upregulated genes in M may act a pivotal part in producing a protective cuticle structure to resist unfavorable conditions (Pesch et al., 2016), steering development, reproduction processes (Grimmelikhuijzen, Cazzamali, Williamson, Schneider, \& Hauser, 2010) and the light detection in reproductive switching (Toyota, Kenji, Sato, \& Tatarazako, 2016). Finally, the upregulated genes in PF compared to SF were mainly enriched in lipid transport, ionotropic glutamate receptor signaling pathway, DNA packaging, transcription, and transferase activity; similarly, the most frequently enriched terms in PF compared to M included DNA packaging, transcription, and lipid transport. These results suggested that the upregulated genes in PF may contribute to maintaining rapid proliferation of parthenogenetic female without fertilization, and nutrient uptake for the growth of neonates (Arbaciauskas, 2004).

In pathway enrichment analysis, we found main stress signaling pathway (PI3K-Akt and MAPK pathways) upregulated in M compared to PF or SF. Interestingly, previous study revealed that these stress signaling 
pathways were activated in response to oxidative stress (Finkel \& Holbrook, 2000). Taken together, these results suggested that the males in D. carinata were more sensitive to environmental stress. Besides, steroid biosynthesis pathway was upregulated in SF compared to PF and M. These results indicated that this pathway might be responsible for the normal development of gonads and oocytes in SF to produce sexual eggs (haploid) requiring fertilization (Banta \& Wood, 2007). Furthermore, basing on the analysis of hub genes and GO/pathway enrichment, several key DEGs involved in reproductive switching were dug out and analyzed with an analogous function as follows.

Cuticle-related genes. Our present study found that several cuticle-related genes, involved in the function of structural constituent of cuticle, were significantly upregulated in SF or M, such as l(3)mbn, dpy-18 , Edg78E , LCP22 , and Lcp65Ab1 were upregulated in SF compared to PF; gbb-1,Lcp65Ab1, Edg78E , and l(3)mbn were upregulated in $\mathrm{M}$ compared to PF; Edg78E , let-653 , Lcp65Ab1 andLCP22 were upregulated in M compared to SF. l(3)mbn and Lcp65Ab1 were cuticle protein genes in Drosophila melanogasterand involved in the formation and development of chitin-based cuticle (Charles, Chihara, Nejad, \& Riddiford, 1997; Karouzou et al., 2007). Similarly, let-653 was related to structural constituent of cuticle in Caenorhabditis elegans (Bird, 1992). Besides, LCP22, gbb-1 and Edg78E were also involved in the formation of cuticle in Bombyx mori and D. melanogaster (Karouzou et al., 2007; Nakato, Shofuda, Izumi, \& Tomino, 1994). The cuticle of arthropods is a composite of chitin and cuticular proteins, which is the most important barrier against the environmental stresses (Karouzou et al., 2007; Liu et al., 2014; Neville, Parry, \& Woodhead-Galloway, 1976; Repka, Walls, \& Ketola, 1995). In cladocerans, the cuticle also consists of cuticle protein and chitin, which can withstand adverse conditions of external environment (Liu et al., 2014; Repka, Walls, \& Ketola, 1995). A recent study demonstrated that D. magna could develop an array of morphological changes in the presence of Triops cancriformis, including changes of carapace morphology and cuticle hardening (Otte, Frohlich, Arnold, \& Laforsch, 2014). Previous studies reported that adverse environmental conditions such as low or high temperature and high population densities could induce the development of males and convert the mode of reproduction to sexual reproduction (Baer \& Owens, 1999; Cao, Lin, \& Guo, 2001; Gordo, Lubian, \& Canavate, 1994). Taken together, the high expression of cuticlerelated genes indicates that these genes could help SF and M to undergo a series of corresponding changes in cuticle structure as a response to the adverse external environmental conditions. Furthermore, the SF needs to produce resting eggs to survive in unfavorable environmental conditions, and those resting eggs are packed by a black ephippium with a thick cuticle. These findings suggest that the up-regulated cuticle-related genes in SF might also play a key role in the formation of ephippium.

Hypoxia-response related genes. In the present study, the globin members (dhb3,APZ42_028671 (Hemoglobin-1), and dhb2) and heme members (Gyc89Da and Gyc89Db) in SF were found to be upregulated compared to PF or M. The globins were a superfamily of heme-containing globular proteins, involved in binding and/or transporting oxygen, specifically for iron ion binding, heme binding, and oxygen transport (Baldi, Chauvin, Hunkapiller, \& Mcclure, 1994; Tavill, Grayzel, London, Williams, \& Vanderhoff, 1968).. Besides, Gyc89Da and Gyc89Db , a guanylyl cyclase, were supposed as oxygen detectors to mediate behavioral responses to hypoxia in Drosophila (Vermehren-Schmaedick, Ainsley, Johnson, Davies, \& Morton, 2010). As we know, environmental signals could regulate a variety of key physiology processes in animals, daphnia could increase hemoglobin production to allow survive in hypoxic stressful environmental condition (Pirow et al., 2001), the hypoxia-response related genes were all up-regulated in SF. These results, taken together, indicate that low oxygen content may lead to reproductive switching of $D$. carinata. On the other hand, the high expression levels of the globin members gene and heme members gene in SF may also point out that SF needs more oxygen to finish sexual reproduction and to produce resting eggs. Given that environmental oxygen levels are relatively low in the occurrence of sexual reproduction in cladoceran, it is further demonstrated that the dissolved oxygen levels may be an important environmental factor for the reproductive switching of cladoceran.

Vitellogenin family genes. Vitellogenin (vg) is an egg yolk (vitellins (Vn)) precursor protein expressed in the females of nearly all oviparous species, including fish, amphibians, reptiles, birds, and most invertebrates (Robinson, 2008). Vitellogenins were a source of nutrients during the early development of oviparous 
invertebrates (Avarre, Lubzens, \& Babin, 2007; Matozzo, Gagne, Marin, Ricciardi, \& Blaise, 2008). The current study found that several vitellogenin family genes were upregulated in PF compared to SF or M, including APZ42_012836 (Apolipophorins ), Vg, VG2, vit-3, vit-6, dmagvtg1 and dmagvtg2 , and they are all annotated as lipid transport. The previous study has reported that the precursor of $\mathrm{Vg}$ (dmagvtg1 and dmagvtg2 ) were the most abundant polypeptide in D. magna eggs (Kato, Tokishita, Ohta, \& Yamagata, 2004; Tokishita et al., 2006). It can thus be suggested that these genes should be involved in the synthesis of egg-yolk proteins in PF to produce more vitellins to package into eggs for the rapid expansion of the population.

Reproduction-related genes. The present study found that several reproduction-related genes were significantly upregulated in SF, such as let-767, ken, cact, Eip 74EF, andl(2)efl were upregulated in SF compared to PF, andFs(2)Ket, RfC4, Ddx1, and $y l$ were upregulated in SF compared to M. l(2)efl belongs to heat shock protein Hsp20 family known as small heat shock proteins (sHSPs), which could resist a harmful stimulus for organisms and participate in the reproduction, development, and normal metabolism activities (Li, Z. W. et al., 2009). Interestingly, some genes related to the development of reproductive system were found to be upregulated in SF, in which let- 767 andken play a key role in female genitalia development in C. elegans and D. melanogaster, respectively (Kuervers, Jones, O'Neil, \& Baillie, 2003; Lukacsovich et al., 2003). Besides, cact ,Eip 74EF , Ddx1 and $y l$ have a function in oogenesis in D. melanogaster (Germain et al., 2015; Kozlova \& Thummel, 2000; Mahowald, 2001; Schupbach \& Wieschaus, 1991), and RfC4 was involved in female meiosis chromosome segregation in D. melanogaster (Dobie et al., 2001). These results suggested that these upregulated genes in SF might also play an important role in female genitalia development and oogenesis in SF for sexual reproduction.

Furthermore, several male trait-related genes were significantly upregulated in M, especially DapmaDsx2, and $p A b p$ upregulated in M compared to PF, and fox-1, DapmaDsx2, and DapmaDsx1-a in M compared to SF. DMRT1 was conserved in a wide range of animals with diverse sex-determining mechanisms (Smith, McClive, Western, Reed, \& Sinclair, 1999), including C. elegans (Raymond et al., 1998),Drosophila (Nothiger et al., 1987), fish (Guan, Kobayashi, \& Nagahama, 2000), reptiles, birds, and mammals (Raymond, Kettlewell, Hirsch, Bardwell, \& Zarkower, 1999). The doublesex (dsx ) has shown deep conservation between Daphnia and genetic sex-determining insect species (Glenner, Thomsen, Hebsgaard, Sørensen, \& Willerslev, 2019), and DapmaDsx1 have been reported inD. magna, which was responsible for the male trait development during environmental sex determination (Kato et al., 2011). Here theDapmaDsx1-a, one of mRNA in DapmaDsx1 encodes protein, showed sex-biased expression in D. carinata males, which suggested that DapmaDsx1-a might be a regulator of the male trait and played a crucial role in sex determination in $D$. carinataduring reproductive switching. Nevertheless, the specific function of another doublesex gene DapmaDsx2 remains unknown (Toyota, K. et al., 2013). Analogously, we also found fox-1 gene, involved in C. elegans primary sex determination (Gladden \& Meyer, 2007), has a sex-biased expression in D. carinata males. Therefore,fox-1 may be a molecule element involved in sex determination in $D$. carinata. Besides, $p A b p$ was participated in spermatogenesis and male meiosis cytokinesis in D. melanogaste(Pertceva et al., 2010), which suggested that $p A b p s$ might be involved in the production of male gametes of $D$. carinata for sexual reproduction.

Methyl farnesoate pathway-related genes. Methyl farnesoate has been associated with a variety of physiological processes in crustaceans related to reproduction, including testicular maturation, ovarian development and mating behavior (Kalavathy, Mamatha, \& Reddy, 1999; Laufer, Ahl, \& Sagi, 1993; Reddy \& Ramamurthi, 1998). In this study, several genes related to the synthesis of methyl farnesoate were found in D. carinata . N-methyl-D-aspartic acid receptors (NMDARs; a type of ionotropic glutamate receptor) likely act as upstream regulators of methyl farnesoate signaling, and was essential for male offspring production (Toyota, K., Miyakawa, Yamaguchi, et al., 2015). In our study, Nmdar1 was found to be upregulated in PF or M compared to SF. In addition, farnesoic acid O-methyltransferase (JHAMT ) was found upregulated in PF compared to SF. JHAMT was the key regulator in methyl farnesoate biosynthesis, which could generate methyl farnesoate by catalyzing farnesoic acid in D. pulex (Huylmans, Ezquerra, Parsch, \& Cordellier, 2016; Toyota, K., Miyakawa, Hiruta, et al., 2015). According to these results, we infer that Nmdar1 andJHAMT in PF could be responsible for methyl farnesoate synthesis to regulate ovarian development in a suitable 
environment or produce male under the adverse environment, and Nmdar1 in M could be responsible for methyl farnesoate synthesis to regulate testicular maturation to make preparations for sexual reproduction.

In insects, methyl farnesoate could be further converted to juvenile hormones by CYP15A1(Li, X. Y., 2007), and juvenile hormones play a key role in regulating development, metamorphosis, and reproduction (DeKort \& Granger, 1996). Previous studies reported that CYP15A1 ortholog was lost in D. pulex genome (Miyakawa, Toyota, \& Sumiya, 2014), but it was detected in our D. carinatagenome (Supplemental Figure S8). These results indicated that methyl farnesoate could not only directly regulate the male sex determine genes, but also be converted to juvenile hormones to regulate these sex determine genes in daphnia. In the present study, CYP15A1 was upregulated in PF compared to SF, and CYP15A1 was upregulated in M compared to PF or SF. We speculated that upregulated CYP15A1 in PF and M could convert methyl farnesoate to juvenile hormones, then juvenile hormones might participate in male sex determine (Figure 11). Furthermore, the detection of CYP15A1 in daphnia could provide a resource for further study in its function and diversity.

\section{miRNAs in reproductive switching biology in $D$. carinata}

In those conserved miRNAs, some reproduction-related miRNA family, such as bantam (Boulan, Martin, \& Milan, 2013), mir-iab-4 (Ronshaugen, Biemar, Piel, Levine, \& Lai, 2005), miR-7 (Lasko, 2011), and miR-252 (Otte, Frohlich, Arnold, \& Laforsch, 2014), were also detected in D. carinata . Hence, it could conceivably be hypothesized that those miRNAs might also play a role in a complex regulatory network of reproductive switching in $D$. carinata. Secondly, in these miRNA/mRNA regulatory modes, the novel-miR-21-lin-9 pairs in M,lin-9 has been reported to play a role in gonad development in C. elegans (Beitel, Lambie, \& Horvitz, 2000). Besides, for the novel-miR-4 - brat and - eIF3-S9 in M, previous studies have demonstrated that brat and $e I F 3-S 9$ were involved in oogenesis (Schupbach \& Wieschaus, 1991) and ovarian follicle cell development (Jia, D. Y. et al., 2015) in D. melanogaster , respectively. These results, taken together, suggested that novelmiR-21 - lin-9, novel-miR-4 - eIF3-S9 and novel-miR-4 - brat regulatory modes might also be involved in reproductive switching in $D$. carinata .

Finally, some upstream transcription regulators of miRNA were found to be involved in the compound eye development in $D$. melanogaster , such as $\operatorname{Kr}$ (Baker et al., 1992), B-H2 (Higashijima et al. 1992), caup(Tomlinson, 2003), exd (Pai et al., 1998), hth (Pichaud \& Casares, 2000), Optix and so (Weasner, Salzer, \& Kumar, 2007). Previous studies have demonstrated that photoreceptors played a key role in resting stage in D. magna (Roulin, Bourgeois, Stiefel, Walser, \& Ebert, 2016) As we know, the resting was a key phenotype in the cyclical parthenogernesis in daphnia. Therefore, these specific upstream transcription regulators might also play an important role in resting in $D$. carinata during reproductive switching.

\section{Global methylation and differentially methylated compare analysis}

Epigenetic modifications have been found to play an important role in environmental stress response in daphnia (Hearn et al., 2019; Jeremias et al., 2018). Explore the DNA methylome of Daphnia with different reproductive status to understand the epigenetic regulation of reproductive switching that contributes to get a new understanding. In this study, the $D$. carinata displayed a low methylation $(1.17 \%)$, which was similar to that reported in other Daphnia spp.(Asselman et al., 2016; Kvist et al., 2018). Previous studies have demonstrated that DNA methylation level was positively correlated with genome size (Ausin et al., 2016; Lechner et al., 2013). The low methylation level in D. carinata maybe due to its small genome size (131.59 Mb).

Even so, compare analysis showed that there were small methylation differences among SF (1.17\%), PF $(1.19 \%)$, and $\mathrm{M}(1.16 \%)$ in D. carinata. The higher methylation level in PF might contribute to its rapid environment stress response (Jeremias et al., 2018). Besides, the patterns of DNA methylation in gene or promoter regions regulating gene transcription in D. carinat were explored, which displayed various forms. 
Only a few genes were displayed with a positive or negative correlation between methylation level and mRNA expression, and positive and negative correlation are both presents in gene body or promotor regions. Consequently, the functional relevant of DMGs and DEGs was confusing in Daphnia. Interestingly, vitelline membrane outer layer protein I-like protein, a noteworthy DMG (promotor region), was more abundantly detected in SF compared to M or PF. Vitelline membrane outer layer protein I (VMO-I) played an important role in the outer layer of the vitelline membrane of eggs (Shimizu, G., Kido, Doi, \& Morikawa, 1994).The highly expressed level of VMO-I like protein in SF indicated that it might be related in the information of a special protective structure of the egg within ephippium.

\section{Acknowledgments}

This article is dedicated to Weimin Wang and Dapeng Li (College of Fisheries, Huazhong Agricultural University) for their genuine interest in training young scientists.

\section{References}

Arbaciauskas, K. (2004). Life-history characteristics and fitness in descendents of parthenogenetic and ex-ephippio females of Daphnia magna .Hydrobiologia, 526 (1), 211-218. doi:https://doi.org/10.1023/b:hydr.0000041602.62586.47

Asselman, J., De Coninck, D. I. M., Pfrender, M. E., \& De Schamphelaere, K. A. C. (2016). Gene Body Methylation Patterns in Daphnia Are Associated with Gene Family Size. Genome Biology and Evolution, 8 (4), 1185-1196. doi:https://doi.org/10.1093/gbe/evw069

Athanasio, C. G., Sommer, U., Viant, M. R., Chipman, J. K., \& Mirbahai, L. (2018). Use of 5azacytidine in a proof-of-concept study to evaluate the impact of pre-natal and post-natal exposures, as well as within generation persistent DNA methylation changes in Daphnia .Ecotoxicology, 27 (5), 556-568. doi:https://doi.org/10.1007/s10646-018-1927-3

Ausin, I., Feng, S. H., Yu, C. W., Liu, W. L., Kuo, H. Y., Jacobsen, E. L., . . Wang, H. F. (2016). DNA methylome of the 20-gigabaseNorway spruce genome. Proceedings of the National Academy of Sciences of the United States of America, 113 (50), E8106-E8113. doi:https://doi.org/10.1073/pnas.1618019113

Avarre, J. C., Lubzens, E., \& Babin, P. J. (2007). Apolipocrustacein, formerly vitellogenin, is the major egg yolk precursor protein in decapod crustaceans and is homologous to insect apolipophorin II/I and vertebrate apolipoprotein B. Bmc Evolutionary Biology, 7 .

Baer, K. N., \& Owens, K. D. (1999). Evaluation of selected endocrine disrupting compounds on sex determination in Daphnia magna using reduced photoperiod and different feeding rates. Bulletin of Environmental Contamination and Toxicology, 62 (2), 214-221. doi:https://doi.org/10.1038/35618

Baker, N. E., Moses, K., Nakahara, D., Ellis, M. C., Carthew, R. W., \& Rubin, G. M. (1992). Mutations on the second chromosome affecting the Drosophila eye. J Neurogenet, 8 (0167-7063 (Print)), 85-100. doi:https://doi.org/10.3109/01677069209084154

Baldi, P., Chauvin, Y., Hunkapiller, T., \& Mcclure, M. A. (1994). Hidden Markov-Models of Biological Primary Sequence Information.Proceedings of the National Academy of Sciences of the United States of America, 91 (3), 1059-1063.

Banta, A., \& Wood, T. (2007). Inheritance in Parthenogenesis and in Sexual Reproduction in Cladocera. Internationale Revue der gesamten Hydrobiologie und Hydrographie, 19 , 264-269. doi:https://doi.org/10.1002/iroh.19280190304

Bao, W. D., Kojima, K. K., $E$ Kohany, O. (2015). Repbase Update, a database of repetitive elements in eukaryotic genomes. Mobile DNA, 6 . 
Beitel, G. J., Lambie, E. J., \& Horvitz, H. R. (2000). The Celegans gene lin-9, which acts in an Rb-related pathway, is required for gonadal sheath cell development and encodes a novel protein. Gene, 254 (1-2), 253-263. doi:https://doi.org/10.1016/s0378-1119(00)00296-1

Bird, D. M. (1992). Sequence Comparison of theCaenorhabditis-Elegans Dpy-13 and Col-34 Genes, and Their Deduced Collagen Products. Gene, 120 (2), 261-266.

Boulan, L., Martin, D., \& Milan, M. (2013). bantam miRNA Promotes Systemic Growth by Connecting Insulin Signaling and Ecdysone Production.Current Biology, 23 (6), 473-478. doi:https://doi.org/10.1016/j.cub.2013.01.072

Burton, J. N., Adey, A., Patwardhan, R. P., Qiu, R. L., Kitzman, J. O., \& Shendure, J. (2013). Chromosomescale scaffolding of de novo genome assemblies based on chromatin interactions. Nature Biotechnology, 31 (12), 1119-+. doi:https://doi.org/10.1038/nbt.2727

Cao, W. Q., Lin, Y. S., \& Guo, D. H. (2001). Effect studies of some environmental conditions on reproductive conversion of Moina mongolica, a salt water cladocera. J. Oceanogr. Taiwan Strait, 20 , 190-195.

Charles, J. P., Chihara, C., Nejad, S., \& Riddiford, L. M. (1997). A cluster of cuticle protein genes of Drosophila melanogaster at 65A: Sequence, structure and evolution. Genetics, 147 (3), 1213-1224.

Colbourne, J. K., Pfrender, M. E., Gilbert, D., Thomas, W. K., Tucker, A., Oakley, T. H., . . Boore, J. L. (2011). The Ecoresponsive Genome of Daphnia pulex . Science, 331 (6017), 555-561.

Colbourne, J. K., Singan, V. R., \& Gilbert, D. G. (2005). wFleaBase: The Daphnia genome database. Bmc Bioinformatics, 6 .

DeKort, C. A. D., \& Granger, N. A. (1996). Regulation of JH titers: The relevance of degradative enzymes and binding proteins. Archives of Insect Biochemistry and Physiology, 33 (1), 1-26. doi:https://doi.org/10.1002/(sici)1520-6327(1996)33:1<1::aid-arch1>3.0.co;2-2

Dinh, H. D. K., Tran, T. H. N., Lu, T. L., Nghiep, T. H., Le, P. N., \& Chi, H. L. (2018). The effect of food, light intensity and tank volume on resting eggs production in Daphnia carinata. Journal of Environmental Management, 217 , 226-230. doi:https://doi.org/10.1016/j.jenvman.2018.03.098

Dobie, K. W., Kennedy, C. D., Velasco, V. M., McGrath, T. L., Weko, J., Patterson, R. W., \& Karpen, G. H. (2001). Identification of chromosome inheritance modifiers in Drosophila melanogaster . Genetics, 157 (4), 1623-1637.

Enright, A. J., John, B., Gaul, U., Tuschl, T., Sander, C., \& Marks, D. S. (2004). MicroRNA targets in Drosophila . Genome Biology, 5 (1).

Feng, H., Conneely, K. N., \& Wu, H. (2014). A Bayesian hierarchical model to detect differentially methylated loci from single nucleotide resolution sequencing data. Nucleic Acids Research, 42 (8). doi:https://doi.org/10.1093/nar/gku154

Finkel, T., \& Holbrook, N. J. (2000). Oxidants, oxidative stress and the biology of ageing. Nature, 408 (6809), 239-247. doi:https://doi.org/10.1038/35041687

Friedlander, M. R., Mackowiak, S. D., Li, N., Chen, W., \& Rajewsky, N. (2012). miRDeep2 accurately identifies known and hundreds of novel microRNA genes in seven animal clades. Nucleic Acids Research, 40 (1), 37-52. doi:https://doi.org/10.1093/nar/gkr688

Genetics, D., \& Maher, E. (2011). DNA methylation: A form of epigenetic control of gene expression. The Obstetrician 83 Gynaecologist, 12 , 37-42. doi:https://doi.org/10.1576/toag.12.1.037.27556

Geng, X. X., Cheng, R. X., Deng, D. G., \& Zhang, H. J. (2016). The complete mitochondrial DNA genome of Chinese Daphnia carinata(Clasocera: Daphniidae). Mitochondrial DNA Part B-Resources, 1 , 323-325. doi:https://doi.org/10.1080/23802359.2016.1172045 
Germain, D. R., Li, L., Hildebrandt, M. R., Simmonds, A. J., Hughes, S. C., \& Godbout, R. (2015). Loss of the Drosophila melanogaster DEAD box protein Ddx1 leads to reduced size and aberrant gametogenesis.Developmental Biology, 407 (2), 232-245. doi:https://doi.org/10.1016/j.ydbio.2015.09.012

Gladden, J. M., \& Meyer, B. J. (2007). A ONECUT homeodomain protein communicates X chromosome dose to specify Caenorhabditis elegans sexual fate by repressing a sex switch gene. Genetics, 177 (0016-6731 (Print)), 1621-1637. doi:https://doi.org/10.1534/genetics.106.061812

Glenner, H., Thomsen, P., Hebsgaard, M., Sørensen, M., \& Willerslev, E. (2019). The origin of insects. Science, $314,1883-1884$.

Gordo, T., Lubian, L. M., \& Canavate, J. P. (1994). Influence of Temperature on Growth, Reproduction and Longevity of Moina-Salina Daday, 1888 (Cladocera, Moinidae). Journal of Plankton Research, 16 (11), 1513-1523. doi:https://doi.org/10.1093/plankt/16.11.1513

Grimmelikhuijzen, C., Cazzamali, G., Williamson, M., Schneider, M., \& Hauser, F. (2010). Invertebrate Neurohormone GPCRs. Encyclopedia of Neuroscience, 205-212. doi:https://doi.org/10.1016/b978-008045046$9.01445-5$

Guan, G., Kobayashi, T., \& Nagahama, Y. (2000). Sexually dimorphic expression of two types of DM (Doublesex/Mab-3)-domain genes in a teleost fish, the Tilapia (Oreochromis niloticus )Biochemical and Biophysical Research Communications, 277 (3), 781-781. doi:https://doi.org/10.1006/bbrc.2000.3713

Haas, B. J., Salzberg, S. L., Zhu, W., Pertea, M., Allen, J. E., Orvis, J., . . Wortman, J. R. (2008). Automated eukaryotic gene structure annotation using EVidenceModeler and the program to assemble spliced alignments. Genome Biology, 9 (1). doi:https://doi.org/10.1186/gb-2008-9-1-r7

Hearn, J., Pearson, M., Blaxter, M., Wilson, P. J., \& Little, T. J. (2019). Genome-wide methylation is modified by caloric restriction inDaphnia magna . BMC Genomics, 20 . doi:https://doi.org/10.1101/278408

Huylmans, A. K., Ezquerra, A. L., Parsch, J., \& Cordellier, M. (2016). De Novo Transcriptome Assembly and Sex-Biased Gene Expression in the Cyclical Parthenogenetic Daphnia galeata. Genome Biology and Evolution, 8 (10), 3120-3139. doi:https://doi.org/10.1093/gbe/evw221

Imakaev, M., Fudenberg, G., McCord, R. P., Naumova, N., Goloborodko, A., Lajoie, B. R., . . . Mirny, L. A. (2012). Iterative correction of Hi-C data reveals hallmarks of chromosome organization. Nature Methods, 9 (10), 999-+. doi:https://doi.org/10.1038/nmeth.2148

Jeremias, G., Barbosa, J., Marques, S. M., De Schamphelaere, K. A. C., Van Nieuwerburgh, F., Deforce, D., . . A Asselman, J. (2018). Transgenerational Inheritance of DNA Hypomethylation in Daphnia magna in Response to Salinity Stress. Environmental Science \& Technology, 52 (17), 10114-10123. doi:https://doi.org/10.1021/acs.est.8b03225

Jia, D. Y., Soylemez, M., Calvin, G., Bornmann, R., Bryant, J., Hanna, C., . . . Deng, W. M. (2015). A large-scale in vivo RNAi screen to identify genes involved in Notch-mediated follicle cell differentiation and cell cycle switches. Scientific Reports, 5 . doi:https://doi.org/10.1038/srep12328

Jia, J. Y., Liu, X. J., Li, L., Lei, C. Q., Dong, Y., Wu, G. Q., \& Hu, G. F. (2018). Transcriptional and Translational Relationship in Environmental Stress: RNAseq and iTRAQ Proteomic Analysis Between Sexually Reproducing and Parthenogenetic Females in Moina micrura . Frontiers in Physiology, 9 , 812. doi:https://doi.org/10.3389/fphys.2018.00812

Jiang, X. D., Li, Q. M., Zhao, S. Y., Zhang, L. H., Zhao, Y. L., Chen, L. Q., . . Liang, H. S. (2014). Temperature reaction norms of Daphnia carinata fitness: the effects of food concentration, population density, and photoperiod. Journal of Freshwater Ecology, 29 (1), 25-35. doi:https://doi.org/10.1080/02705060.2013.823467

Kalavathy, Y., Mamatha, P., \& Reddy, P. S. (1999). Methyl farnesoate stimulates testicular growth in the freshwater crab Oziotelphusa senex senex fabricius. Naturwissenschaften, 86 (8), 394-395. 
Karouzou, M. V., Spyropoulos, Y., Iconomidou, V. A., Cornman, R. S., Hamodrakas, S. J., \& Willis, J. H. (2007). Drosophila cuticular proteins with the R\&R Consensus: Annotation and classification with a new tool for discriminating RR-1 and RR-2 sequences. Insect Biochemistry and Molecular Biology, 37 (8), 754-760. doi:https://doi.org/10.1016/j.ibmb.2007.03.007

Kato, Y., Kobayashi, K., Oda, S., Colbourn, J. K., Tatarazako, N., Watanabe, H., \& Iguchi, T. (2008). Molecular cloning and sexually dimorphic expression of DM-domain genes in Daphnia magna .Genomics, 91 (1), 94-101. doi:https://doi.org/10.1016/j.ygeno.2007.09.002

Kato, Y., Kobayashi, K., Oda, S., Tatarazako, N., Watanabe, H., \& Iguchi, T. (2010). Sequence divergence and expression of a transformer gene in the branchiopod crustacean, Daphnia magna .Genomics, 95 (3), 160-165. doi:https://doi.org/10.1016/j.ygeno.2009.12.005

Kato, Y., Kobayashi, K., Watanabe, H., \& Iguchi, T. (2011). Environmental Sex Determination in the Branchiopod CrustaceanDaphnia magna : Deep Conservation of a Doublesex Gene in the Sex-Determining Pathway. Plos Genetics, 7 (3). doi:https://doi.org/10.1371/journal.pgen.1001345

Kato, Y., Tokishita, S., Ohta, T., \& Yamagata, H. (2004). A vitellogenin chain containing a superoxide dismutase-like domain is the major component of yolk proteins in cladoceran crustacean Daphnia magna . Gene, 334, 157-165. doi:https://doi.org/10.1016/j.gene.2004.03.030

Kim, H., Koedrith, P., \& Seo, Y. (2015). Ecotoxicogenomic Approaches for Understanding Molecular Mechanisms of Environmental Chemical Toxicity Using Aquatic Invertebrate, Daphnia Model Organism.International Journal of Molecular Sciences, 16 , 12261-12287. doi:https://doi.org/10.3390/ijms160612261

Kong, L., Li, H. X., Wu, D. L., Xu, G. R., Wang, D. L., \& Zhao, Y. L. (2016). Molecular characterization of the gene checkpoint homolog 1 inDaphnia carinata during different reproductive phases. Genetics and Molecular Research, 15 (2). doi:https://doi.org/10.4238/gmr.15026132

Kozlova, T., \& Thummel, C. S. (2000). Steroid regulation of postembryonic development and reproduction in Drosophila .Trends in Endocrinology and Metabolism, 11 (7), 276-280. doi:https://doi.org/10.1016/s10432760(00)00282-4

Krueger, F., \& Andrews, S. R. (2011). Bismark: a flexible aligner and methylation caller for Bisulfite-Seq applications. Bioinformatics, 27 (11), 1571-1572. doi:https://doi.org/10.1093/bioinformatics/btr167

Kuervers, L., Jones, C., O’Neil, N., \& Baillie, D. (2003). The sterol modifying enzyme LET-767 is essential for growth, reproduction and development in Caenorhabditis elegans. Molecular genetics and genomics : MGG, 270 , 121-131. doi:https://doi.org/10.1007/s00438-003-0900-9

Kvist, J., Athanasio, C. G., Solari, O. S., Brown, J. B., Colbourne, J. K., Pfrender, M. E., \& Mirbahai, L. (2018). Pattern of DNA Methylation inDaphnia : Evolutionary Perspective. Genome Biology and Evolution, 10 (8), 1988-2007. doi:https://doi.org/10.1093/gbe/evy155

Langmead, B., Trapnell, C., Pop, M., \& Salzberg, S. L. (2009). Ultrafast and memory-efficient alignment of short DNA sequences to the human genome. Genome Biology, 10 (3). doi:https://doi.org/10.1186/gb-200910-3-r25

Lasko, P. (2011). Posttranscriptional regulation in Drosophila oocytes and early embryos. Wiley Interdisciplinary Reviews-Rna, 2 (3), 408-416. doi:https://doi.org/10.1002/wrna.70

Laufer, H., Ahl, J. S. B., \& Sagi, A. (1993). The Role of Juvenile Hormones in Crustacean Reproduction. American Zoologist, 33 (3), 365-374. doi:https://doi.org/10.1093/icb/33.3.365

Lechner, M., Marz, M., Ihling, C., Sinz, A., Stadler, P. F., \& Krauss, V. (2013). The correlation of genome size and DNA methylation rate in metazoans. Theory in Biosciences, 132 (1), 47-60. doi:https://doi.org/10.1007/s12064-012-0167-y 
Lee, B. Y., Choi, B. S., Kim, M. S., Park, J. C., Jeong, C. B., Han, J., \& Lee, J. S. (2019). The genome of the freshwater water flea Daphnia magna: A potential use for freshwater molecular ecotoxicology. Aquatic Toxicology, 210 , 69-84. doi:https://doi.org/10.1016/j.aquatox.2019.02.009

Lekamge, S., Miranda, A. F., Ball, A. S., Shukla, R., \& Nugegoda, D. (2019). The toxicity of coated silver nanoparticles to Daphnia carinata and trophic transfer from alga Raphidocelis subcapitata . PLoS One, 14 (4). doi:https://doi.org/10.1371/journal.pone.0214398

Li, X. Y. (2007). Juvenile hormone and methyl farnesoate production in cockroach embryos in relation to dorsal closure and the reproductive modes of different species of cockroaches. Archives of Insect Biochemistry and Physiology, 66 (4), 159-168. doi:https://doi.org/10.1002/arch.20207

Li, Z. W., Li, X., Yu, Q. Y., Xiang, Z. H., Kishino, H., \& Zhang, Z. (2009). The small heat shock protein (sHSP) genes in the silkworm,Bombyx mori, and comparative analysis with other insectsHSP genes. Bmc Evolutionary Biology, 9 . doi:https://doi.org/10.1186/1471-2148-9-215

Liu, A. J., Zhang, M. Q., Kong, L., Wu, D. L., Weng, X. L., Wang, D. L., \& Zhao, Y. L. (2014). Cloning and expression profiling of a cuticular protein gene in Daphnia carinata . Development Genes and Evolution, 224 (3), 129-135. doi:https://doi.org/10.1007/s00427-014-0469-9

Lowe, T. M., \& Eddy, S. R. (1997). tRNAscan-SE: A program for improved detection of transfer RNA genes in genomic sequence. Nucleic Acids Research, 25 (5), 955-964. doi:https://doi.org/10.1093/nar/25.5.0955

Lukacsovich, T., Yuge, K., Awano, W., Asztalos, Z., Kondo, S., Juni, N., \& Yamamoto, D. (2003). The ken and barbie gene encoding A putative transcription factor with a BTB domain and three zinc finger motifs functions in terminalia development of Drosophila. Archives of Insect Biochemistry and Physiology, 54 (2), 77-94. doi:https://doi.org/10.1002/arch.10105

Mahowald, A. P. (2001). Assembly of the Drosophila germ plasm.International Review of Cytology - a Survey of Cell Biology, Vol 203, 203, 187-213. doi:https://doi.org/10.1016/s0074-7696(01)03007-8

Matozzo, V., Gagne, F., Marin, M. G., Ricciardi, F., \& Blaise, C. (2008). Vitellogenin as a biomarker of exposure to estrogenic compounds in aquatic invertebrates: A review. Environment International, 34 (4), 531-545. doi:https://doi.org/10.1016/j.envint.200\%.09.008

Miyakawa, H., Toyota, K., \& Sumiya, E. (2014). Comparison of JH signaling in insects and crustaceans. Current Opinion in Insect Science, 1 . doi:https://doi.org/10.1016/j.cois.2014.04.006

Nakato, H., Shofuda, K., Izumi, S., \& Tomino, S. (1994). Structure and Developmental Expression of a Larval Cuticle Protein Gene of the Silkworm, Bombyx-Mori . Biochimica Et Biophysica Acta-Gene Structure and Expression, 1218 (1), 64-74. doi:https://doi.org/10.1016/0167-4781(94)90101-5

Nawrocki, E. P., \& Eddy, S. R. (2013). Infernal 1.1: 100-fold faster RNA homology searches. Bioinformatics, 29 (22), 2933-2935. doi:https://doi.org/10.1093/bioinformatics/btt509

Neville, A. C., Parry, D., \& Woodhead-Galloway, J. (1976). The chitin crystallite in arthropod cuticle. Journal of cell science, 21, 73-82.

Nothiger, R., Leuthold, M., Andersen, N., Gerschwiler, P., Gruter, A., Keller, W., . . Schmid, H. (1987). Genetic and Developmental Analysis of the Sex-Determining Gene Double Sex (Dsx) ofDrosophila-Melanogaster . Genetical Research, 50 (2), 113-123. doi:https://doi.org/10.1017/s001667230002351x

Olmstead, A. W., \& LeBlanc, G. A. (2003). Insecticidal juvenile hormone analogs stimulate the production of male offspring in the crustaceanDaphnia magna . Environmental Health Perspectives, 111 (7), 919-924. doi:https://doi.org/10.1289/ehp.5982

Otte, K. A., Frohlich, T., Arnold, G. J., \& Laforsch, C. (2014). Proteomic analysis of Daphnia magna hints at molecular pathways involved in defensive plastic responses. BMC Genomics, 15 . doi:https://doi.org/10.1186/1471-2164-15-306 
Pai, C. Y., Kuo, T. S., Jaw, T. J., Kurant, E., Chen, C. T., Bessarab, D. A., . . . Sun, Y. H. (1998). The Homothorax homeoprotein activates the nuclear localization of another homeoprotein, Extradenticle, and suppresses eye development in Drosophila. Genes 83 Development, 12 (3), 435-446. doi:https://doi.org/10.1101/gad.12.3.435

Park, Y., \& Wu, H. (2016). Differential methylation analysis for BS-seq data under general experimental design. Bioinformatics, 32 (10), 1446-1453. doi:https://doi.org/10.1093/bioinformatics/btw026

Pertceva, J. A., Dorogova, N. V., Bolobolova, E. U., Nerusheva, O. O., Fedorova, S. A., \& Omelyanchuk, L. V. (2010). The role of Drosophila hyperplastic discs gene in spermatogenesis. Cell Biology International, 34 (10), 991-996.

Pesch, Y. Y., Riedel, D., Patil, K. R., Loch, G., \& Behr, M. (2016). Chitinases and Imaginal disc growth factors organize the extracellular matrix formation at barrier tissues in insects. Scientific Reports, 6 . doi:https://doi.org/10.1038/srep18340

Pichaud, F., \& Casares, F. (2000). Homothorax and iroquois-C genes are required for the establishment of territories within the developing eye disc. Mechanisms of Development, 96 (1), 15-25. doi:https://doi.org/10.1016/s0925-4773(00)00372-5

Pirow, R., Baumer, C., \& Paul, R. J. (2001). Benefits of haemoglobin in the cladoceran crustacean Daphnia magna . Journal of Experimental Biology, 204 (20), 3425-3441.

Raymond, C. S., Kettlewell, J. R., Hirsch, B., Bardwell, V. J., \& Zarkower, D. (1999). Expression of Dmrt1 in the genital ridge of mouse and chicken embryos suggests a role in vertebrate sexual development.Developmental Biology, 215 (2), 208-220. doi:https://doi.org/10.1006/dbio.1999.9461

Raymond, C. S., Shamu, C. E., Shen, M. M., Seifert, K. J., Hirsch, B., Hodgkin, J., \& Zarkower, D. (1998). Evidence for evolutionary conservation of sex-determining genes. Nature, 391 (6668), 691-695.

Reddy, P. S., \& Ramamurthi, P. (1998). Methyl farnesoate stimulates ovarian maturation in the freshwater crab Oziotelphusa senex senex Fabricius. Current Science, 74 (1), 68-70. doi:https://doi.org/10.1007/s001140050639

Repka, S., Walls, M., \& Ketola, M. (1995). Neck Spine Protects Daphnia-Pulex from Predation by Chaoborus, but Individuals with Longer Tail Spine Are at a Greater Risk. Journal of Plankton Research, 17 (2), 393-403. doi:https://doi.org/10.1093/plankt/17.2.393

Robinson, R. (2008). For Mammals, Loss of Yolk and Gain of Milk Went Hand in Hand. PLoS biology, 6 , e77. doi:https://doi.org/10.1371/journal.pbio.0060077

Ronshaugen, M., Biemar, F., Piel, J., Levine, M., \& Lai, E. C. (2005). The Drosophila microRNA iab-4 causes a dominant homeotic transformation of halteres to wings. Genes $\& 3$ Development, 19 (24), 2947-2952. doi:https://doi.org/10.1101/gad.1372505

Roulin, A. C., Bourgeois, Y., Stiefel, U., Walser, J. C., \& Ebert, D. (2016). A Photoreceptor Contributes to the Natural Variation of Diapause Induction in Daphnia magna . Molecular Biology and Evolution, 33 (12), 3194-3204. doi:https://doi.org/10.1093/molbev/msw200

Schupbach, T., \& Wieschaus, E. (1991). Female Sterile Mutations on the 2nd Chromosome of DrosophilaMelanogaster .2. Mutations Blocking Oogenesis or Altering Egg Morphology. Genetics, 129(4), 1119-1136.

Seberg, O., \& Petersen, G. (2009). A unified classification system for eukaryotic transposable elements should reflect their phylogeny. Nature Reviews Genetics, 10(4), 276-276.

Shannon, P., Markiel, A., Ozier, O., Baliga, N. S., Wang, J. T., Ramage, D., . . Ideker, T. (2003). Cytoscape: A software environment for integrated models of biomolecular interaction networks. Genome Research, 13 (11), 2498-2504. doi:https://doi.org/10.1101/gr.1239303 
Shimizu, T., G., V. D., Kido, S., Doi, Y., \& Morikawa, K. (1994). Crystal structure of vitelline membrane outer layer protein I (VMO-I): a folding motif with homologous Greek key structures related by an internal three-fold symmetry. EMBO J, 13 (0261-4189 (Print)), 1003-1010. doi:https://doi.org/10.1002/j.14602075.1994.tb06348.x

Simao, F. A., Waterhouse, R. M., Ioannidis, P., Kriventseva, E. V., \& Zdobnov, E. M. (2015). BUSCO: assessing genome assembly and annotation completeness with single-copy orthologs. Bioinformatics, 31 (19), 3210-3212. doi:https://doi.org/10.3410/f.725547803.793528083

Smith, C. A., McClive, P. J., Western, P. S., Reed, K. J., \& Sinclair, A. H. (1999). Conservation of a sex-determining gene. Nature, 402 (6762), 601-602. doi:https://doi.org/10.1038/45130

Song, K., Li, L., \& Zhang, G. F. (2017). The association between DNA methylation and exon expression in the Pacific oyster Crassostrea giga s. PLoS One, 12 (9). doi:https://doi.org/10.1371/journal.pone.0185224

Strepetkaite, D., Alzbutas, G., Astromskas, E., Lagunavicius, A., Sabaliauskaite, R., Arbaciauskas, K., \& Lazutka, J. (2016). Analysis of DNA Methylation and Hydroxymethylation in the Genome of CrustaceanDaphnia pulex . Genes, 7 (1). doi:https://doi.org/10.3390/genes7010001

Tatarazako, N., Oda, S., Watanabe, H., Morita, M., \& Iguchi, T. (2003). Juvenile hormone agonists affect the occurrence of male Daphnia.Chemosphere, 53 (8), 827-833. doi:https://doi.org/10.1016/s00456535(03)00761-6

Tavill, A., Grayzel, A., London, I., Williams, M., \& Vanderhoff, G. (1968). The Role of Heme in the Synthesis and Assembly of Hemoglobin. Journal of Biological Chemistry, 243 , 4987-4999.

Tokishita, S., Kato, Y., Kobayashi, T., Nakamura, S., Ohta, T., \& Yamagata, H. (2006). Organization and repression by juvenile hormone of a vitellogenin gene cluster in the crustacean, Daphnia magna .Biochemical and Biophysical Research Communications, 345 (1), 362-370. doi:https://doi.org/10.1016/j.bbrc.2006.04.102

Tomlinson, A. (2003). Patterning the peripheral retina of the fly: Decoding a gradient. Developmental Cell, 5 (5), 799-809. doi:https://doi.org/10.1016/s1534-5807(03)00326-5

Toyota, K., Kato, Y., Sato, M., Sugiura, N., Miyagawa, S., Miyakawa, H., . . . Iguchi, T. (2013). Molecular cloning of doublesex genes of four cladocera (water flea) species. BMC Genomics, 14 . doi:https://doi.org/10.1186/1471-2164-14-239

Toyota, K., Miyakawa, H., Hiruta, C., Furuta, K., Ogino, Y., Shinoda, T., . . Iguchi, T. (2015). Methyl farnesoate synthesis is necessary for the environmental sex determination in the water flea Daphnia pulex . Journal of Insect Physiology, 80 , 22-30. doi:https://doi.org/10.1016/j.jinsphys.2015.02.002

Toyota, K., Miyakawa, H., Yamaguchi, K., Shigenobu, S., Ogino, Y., Tatarazako, N., . . Iguchi, T. (2015). NMDA receptor activation upstream of methyl farnesoate signaling for short day-induced male offspring production in the water flea, Daphnia pulex . BMC Genomics, 16 . doi:https://doi.org/10.1186/s12864-0151392-9

Toyota, K., Sato, T., \& Tatarazako, N. (2016). Protein kinase C is involved with upstream signaling of methyl farnesoate for photoperiod-dependent sex determination in the water flea Daphnia pulex . Biology open, 6 . doi:https://doi.org/10.1242/bio.021857

Trapnell, C., Pachter, L., \& Salzberg, S. L. (2009). TopHat: discovering splice junctions with RNA-Seq. Bioinformatics, 25 (9), 1105-1111. doi:https://doi.org/10.1093/bioinformatics/btp120

Vermehren-Schmaedick, A., Ainsley, J. A., Johnson, W. A., Davies, S. A., \& Morton, D. B. (2010). Behavioral Responses to Hypoxia in Drosophila Larvae Are Mediated by Atypical Soluble Guanylyl Cyclases. Genetics, 186 (1), 183-U317. doi:https://doi.org/10.1534/genetics.110.118166

Wang, X., Wheeler, D., Avery, A., Rago, A., Choi, J. H., Colbourne, J. K., . . Werren, J. H. (2013). Function and Evolution of DNA Methylation in Nasonia vitripennis . Plos Genetics, 9 (10). 
Weasner, B., Salzer, C., \& Kumar, J. P. (2007). Sine oculis, a member of the SIX family of transcription factors, directs eye formation.Developmental Biology, 303 (2), 756-771. doi:https://doi.org/10.1016/j.ydbio.2006.10.040

Wiggins, P. R., \& Frappell, P. B. (2000). The influence of haemoglobin on behavioural thermoregulation and oxygen consumption in Daphnia carinata. Physiological and Biochemical Zoology, 73 (2), 153-160. doi:https://doi.org/10.1086/316739

Wu, H., Xu, T. L., Feng, H., Chen, L., Li, B., Yao, B., . . Conneely, K. N. (2015). Detection of differentially methylated regions from whole-genome bisulfite sequencing data without replicates. Nucleic Acids Research, $43(21)$.

Xiang, H., Zhu, J. D., Chen, Q. A., Dai, F. Y., Li, X., Li, M. W., . . Wang, J. (2010). Single base-resolution methylome of the silkworm reveals a sparse epigenomic map. Nature Biotechnology, 28 (7), 756-756.

Xu, X. Q., Song, S. H., Wang, Q., Qin, F., Liu, K., Zhang, X. W., . . Z Zhao, Y. L. (2009). Analysis and comparison of a set of expressed sequence tags of the parthenogenetic water flea Daphnia carinata.Molecular Genetics and Genomics, 282 (2), 197-203. doi:https://doi.org/10.1007/s00438-009-0459-1

Ye, Z. Q., Xu, S., Spitze, K., Asselman, J., Jiang, X. Q., Ackerman, M. S., . . Lynch, M. (2017). A New Reference Genome Assembly for the Microcrustacean Daphnia pulex . G3-Genes Genomes Genetics, 7 (5), 1405-1416.

Zhang, M. F., Zeng, C., Chen, Y. S., \& Zhao, Y. L. (2009). Karyologic observation on maturation of germ cells of daphnia (ctenodaphnia) carinata . Journal of Fishery Sciences of China, 16 (9), 868-877.

\section{Data Accessibility}

The sequence data supporting the results of this article are available in Sequence Read Archive at NCBI SRP228147, including the Illumina genomic sequencing reads, Pacbio long reads, Hi-C data, DNA methylation data, miRNA data and RNA-seq reads. The chromosome-level assembly was available in the GenBank at NCBI WJBH00000000.

\section{Author contributions}

X.J.L. and G.F.H. conceived the study. J.Y.J. and C.F performed the experiments. C.C.D. and M.Q.H. contributed to the culture of the sample. J.Y.J. conducted the bioinformatics analysis. J.Y.J. wrote the manuscript with assistance from G.F.H.

\section{Funding}

This research was supported by the Fundamental Research Funds for the Central Universities to X.L. (2662019FW007) and G.H. (2662019PY006). The Financial support was also provided from the National Natural Science Foundation of China NSFC (31500374 for X.L.) and Natural Science Foundation of Hubei Province (2019CFB493 for X.L).

\section{Conflict of Interest Statement}

The authors declare that the research was conducted in the absence of any commercial or financial relationships that could be construed as a potential conflict of interest. 


\section{Conflict of interest}

We have no conflict of interest to declare and informed consent was obtained.

\section{Tables}

Table 1 Statistics of the final assemblies of the D. carinata using de nove and Hi-C data

\begin{tabular}{llll}
\hline Items & & Hybrid assembly & Hi-C assembly \\
\hline Contig & Number & 470 & 527 \\
& Size (bp) & $131,583,282$ & $131,583,282$ \\
& N50 (bp) & $3,360,086$ & $2,802,846$ \\
& N90 (bp) & 92,925 & 84,515 \\
& Max (bp) & $7,729,844$ & $6,650,000$ \\
Scaffold & Gap total length (bp) & 0 & 0 \\
& Number & 470 & 454 \\
& Size (bp) & $131,583,282$ & $131,590,582$ \\
& N50 (bp) & $3,360,086$ & $8,414,058$ \\
& N90 (bp) & 92,925 & 88,181 \\
& Max (bp) & $7,729,844$ & $14,956,565$ \\
GC ratio (\%) & Gap total length (bp) & 0 & 7,300 \\
\hline
\end{tabular}

*Hybrid assembly means de novo assembly using Illumina and PacBio data in our study.

Table 2 The noteworthy pathways in SF, PF, and M

\begin{tabular}{|c|c|c|c|}
\hline Pathway & Regulate & $\mathrm{Q}$ value & Genes \\
\hline \multicolumn{4}{|l|}{ SF vs. PF } \\
\hline Glutathione metabolism & Up & $4.63 \mathrm{E}-02$ & APN2, APZ42_022935, ZW, APZ42_033731 \\
\hline Hedgehog signaling pathway & $\mathrm{Up}$ & 4.17E-02 & $r d x$, mel-26 \\
\hline Longevity regulating pathway - multiple species & $\mathrm{Up}$ & $2.34 \mathrm{E}-04$ & SODC, OV25-1, l(2)efl, Hsp23, APZ42_0269 \\
\hline Steroid biosynthesis & $\mathrm{Up}$ & $5.22 \mathrm{E}-03$ & $D D B_{-} G 0269788, D D B_{-} G 0270946$ \\
\hline Glycosphingolipid biosynthesis - globo series & Down & $2.35 \mathrm{E}-03$ & brn \\
\hline Glycosphingolipid biosynthesis - lacto and neolacto & Down & $2.24 \mathrm{E}-02$ & $b r n$ \\
\hline Hedgehog signaling pathway - fly & Down & $2.71 \mathrm{E}-02$ & APZ42_033710, ptc, hh \\
\hline Insect hormone biosynthesis & Down & $3.53 \mathrm{E}-02$ & JHAMT, CYP15A1 \\
\hline \multicolumn{4}{|l|}{ M vs. PF } \\
\hline Phototransduction - fly & Up & $2.39 \mathrm{E}-04$ & Trpgamma, norpA, ninaC, trp, trpl, APZ42_ \\
\hline Neuroactive ligand-receptor interaction & $\mathrm{Up}$ & $3.84 \mathrm{E}-03$ & APZ42_018707, etaTry, AR, TRYP7, mGluh \\
\hline Steroid biosynthesis & $\mathrm{Up}$ & $4.58 \mathrm{E}-02$ & $D D B_{-} G 0270946$ \\
\hline Insect hormone biosynthesis & $\mathrm{Up}$ & $4.69 \mathrm{E}-02$ & CYP15A1 \\
\hline Wnt signaling pathway & $\mathrm{Up}$ & 2.03E-02 & $n o r p A, e b i, w g$ \\
\hline Oocyte meiosis & $\mathrm{Up}$ & $3.81 \mathrm{E}-02$ & $A P Z 42 \_011481$ \\
\hline Spliceosome & Down & $2.56 \mathrm{E}-04$ & APZ42_026804, CG13298, sf3b3, wds, EF2, \\
\hline Pyrimidine metabolism & Down & $1.83 \mathrm{E}-02$ & APZ42_013907, APZ42_021143, CG12018, T \\
\hline FoxO signaling pathway & Down & $2.48 \mathrm{E}-02$ & C02F5.7, APZ42_034302, APZ42_019766, cy \\
\hline Insect hormone biosynthesis & Down & 4.87E-02 & JHAMT, shd \\
\hline
\end{tabular}




\begin{tabular}{llll}
\hline Pathway & Regulate & Q value & Genes \\
\hline Neuroactive ligand-receptor interaction & $\mathrm{Up}$ & $2.64 \mathrm{E}-05$ & APZ42_018707, TRYP7, GluRIA, QRFPR, \\
Phototransduction - fly & $\mathrm{Up}$ & $7.15 \mathrm{E}-03$ & Trpgamma, norpA, ninaC, trp, trpl, APZ42_l \\
Glycosphingolipid biosynthesis-lacto and neolacto & $\mathrm{Up}$ & $2.47 \mathrm{E}-02$ & gnt14 \\
ECM-receptor interaction & $\mathrm{Up}$ & $4.85 \mathrm{E}-02$ & scaf, LanB2, LanA, N \\
Wnt signaling pathway & $\mathrm{Up}$ & $4.22 \mathrm{E}-02$ & norpA, Notum, ebi, wg \\
MAPK signaling pathway & $\mathrm{Up}$ & $4.52 \mathrm{E}-02$ & mkkA \\
Insect hormone biosynthesis & $\mathrm{Up}$ & $4.10 \mathrm{E}-02$ & CYP15A1 \\
Hedgehog signaling pathway & Down & $8.18 \mathrm{E}-05$ & bath-43, mel-26, bath-42 \\
Longevity regulating pathway - multiple species & Down & $6.05 \mathrm{E}-04$ & SODC,APZ42_026804, OV25-1, Sod1, l(2)ef \\
Steroid biosynthesis & Down & $4.97 \mathrm{E}-02$ & DDB_G0269788, Lip1, Lip3 \\
Terpenoid backbone biosynthesis & Down & $3.15 \mathrm{E}-02$ & ipi, coq1, APZ42_011829 \\
Fatty acid elongation & Down & $4.55 \mathrm{E}-02$ & let-767, hpo-8 \\
\hline
\end{tabular}

\section{Figures}

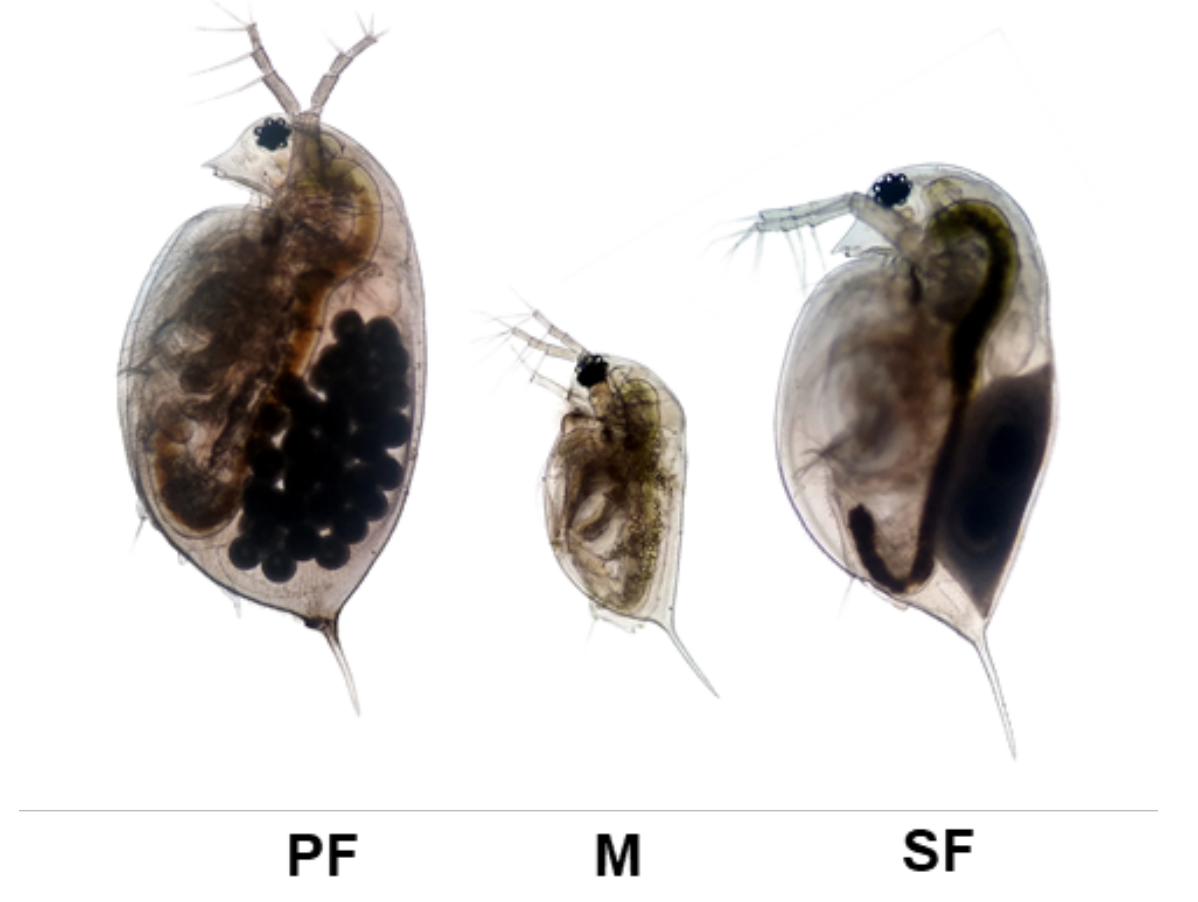

Figure 1 The photograph of Daphnia carinata .

PF: parthenogenetic female; M: male; SF: sexual female. 


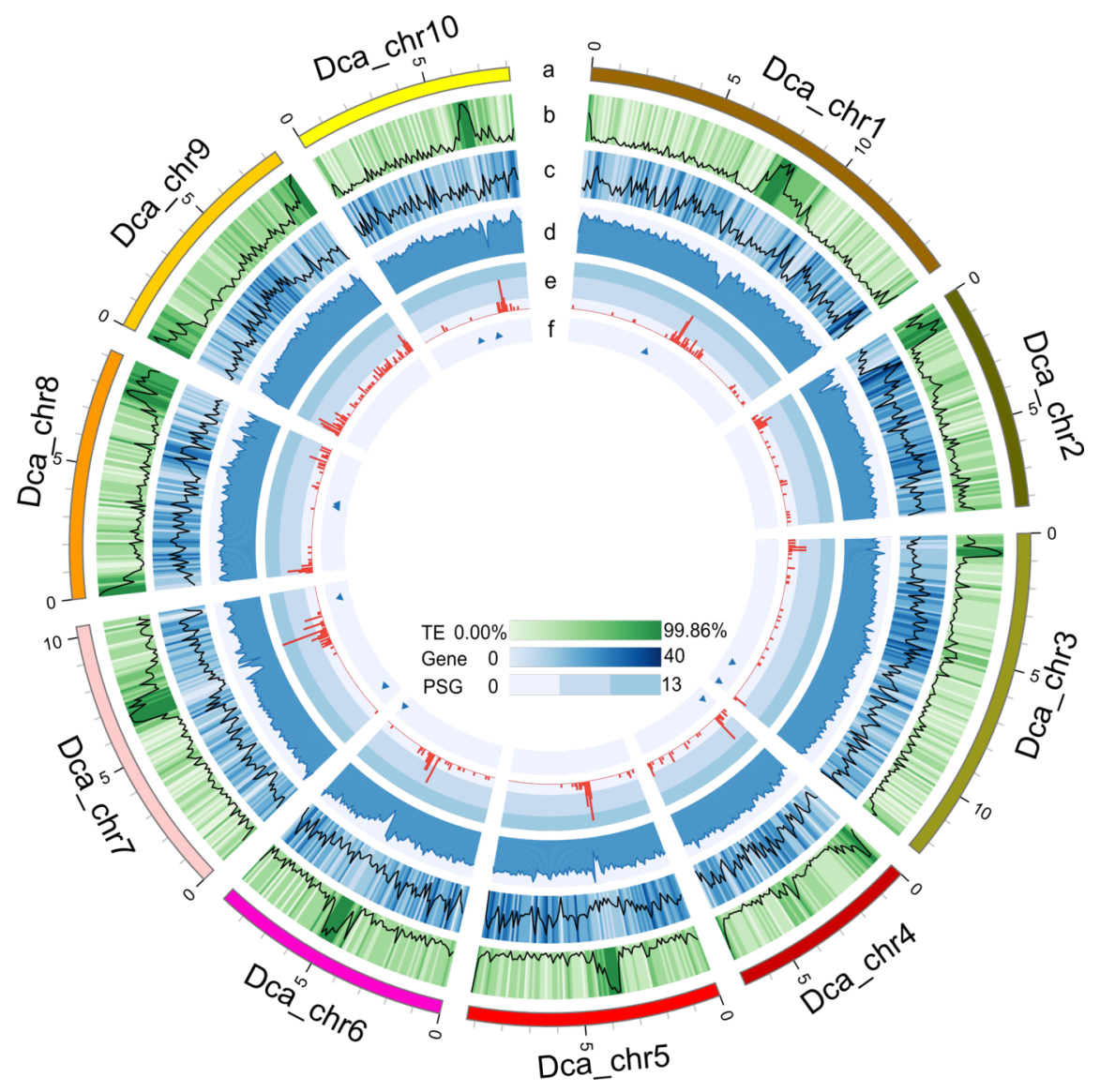

Figure 2 D. carinata genome overview.

Genome features in $100-\mathrm{Kb}$ intervals across the 10 Chromosomes. From outer to inner circles, a: each $D$. carinata chromosome (Dca_chr1-Dca_chr10); b: Transposon elements (TE) coverage (0\%-99.86\% per 100$\mathrm{Kb}$ ); c: Gene density (number per 100-Kb, range 0-40); d: GC contents (30.29\%-44.54\% per 100-Kb); e: Pseudogenes (PSG) density (number per 100-Kb, 0-13); f: distribution of miRNAs in chromosomes. 


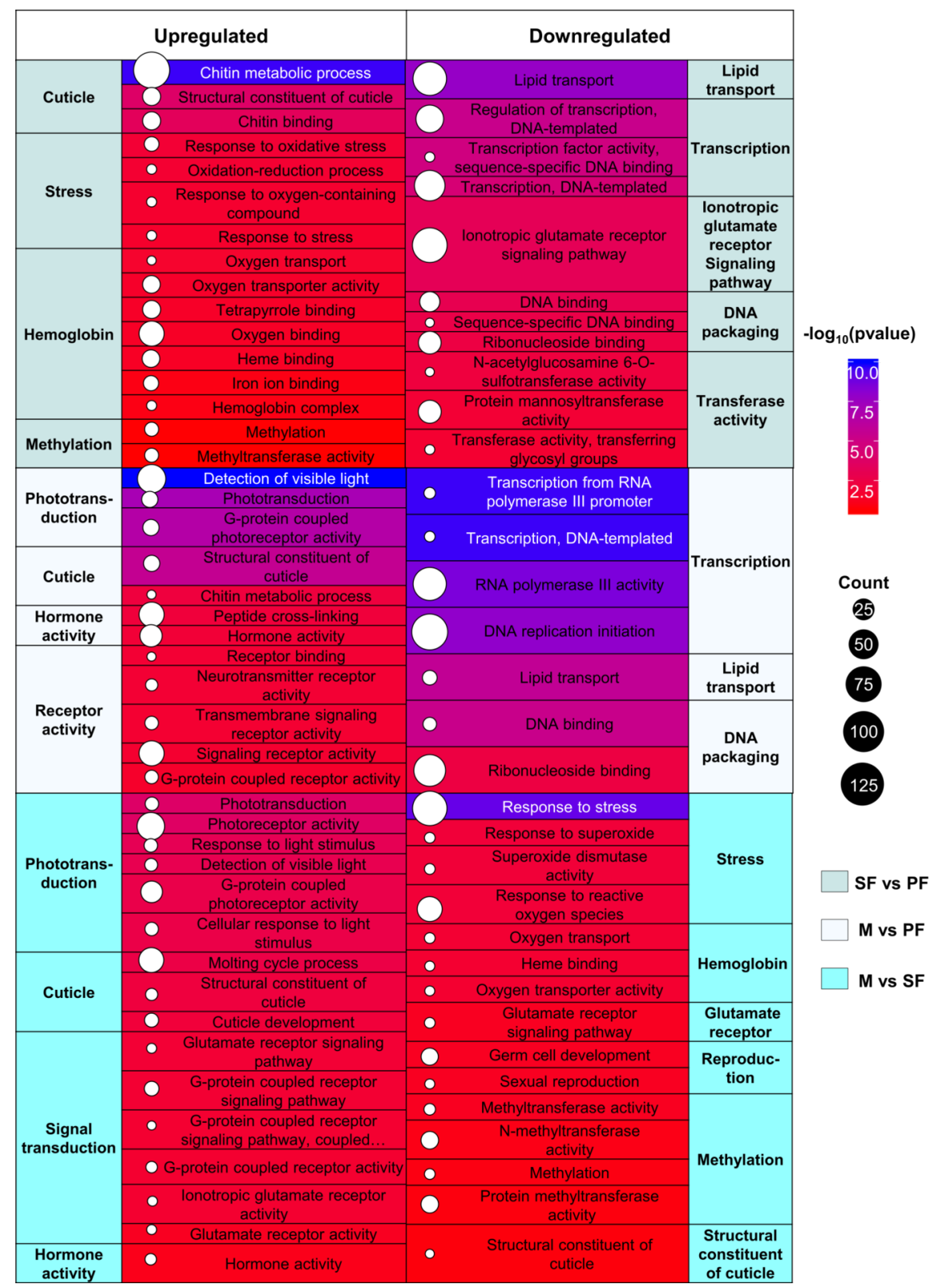

Figure 3 The GO enrichment analysis of DEGs.

The P-values were calculated using the Benjamini-corrected modified Fisher's exact test. 
Up Down Up Down Up Down

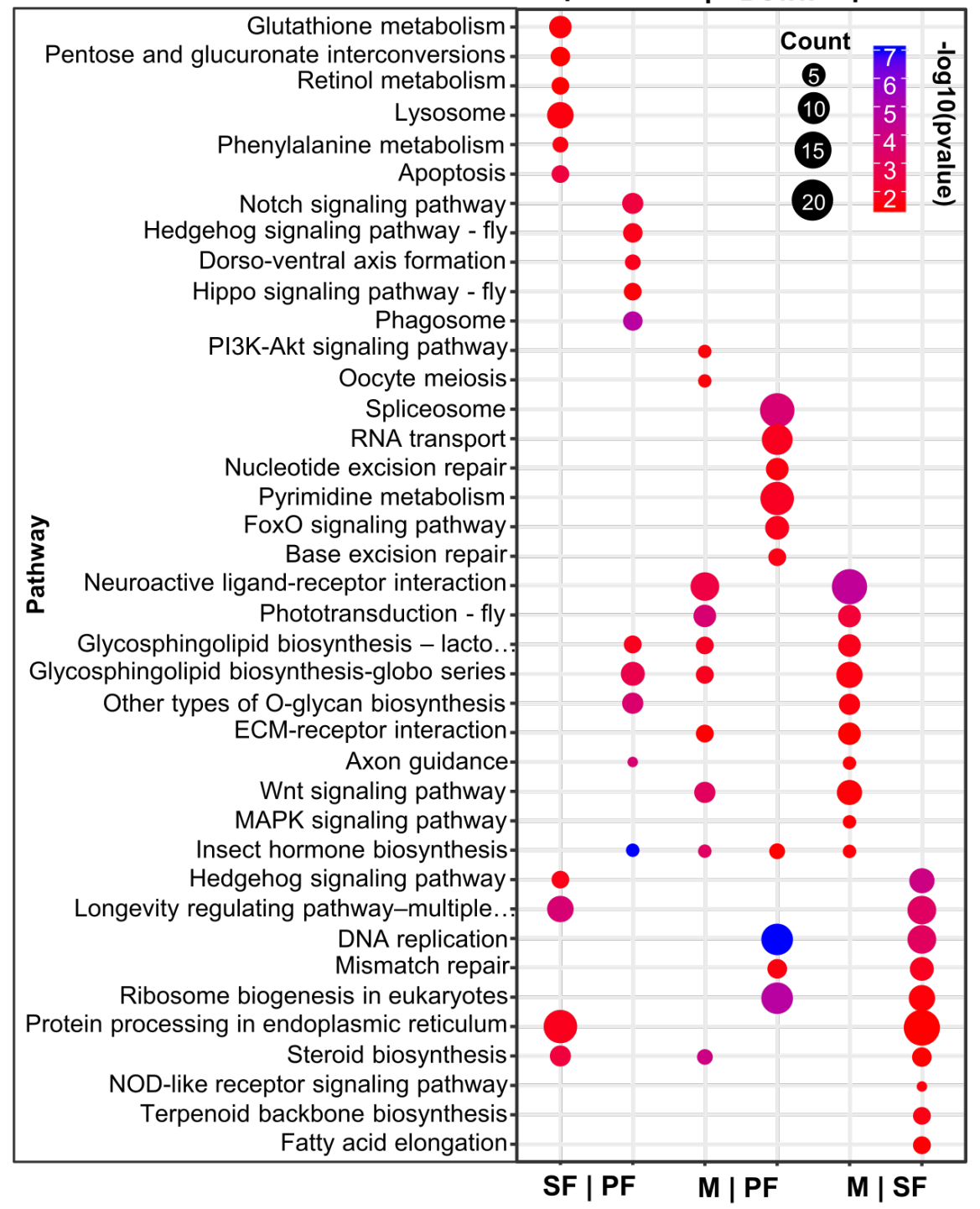

Figure 4 The enriched KEGG pathways of DEGs.

The P-values were calculated using the Benjamini-corrected modified Fisher's exact test. 


\section{Fig.7}

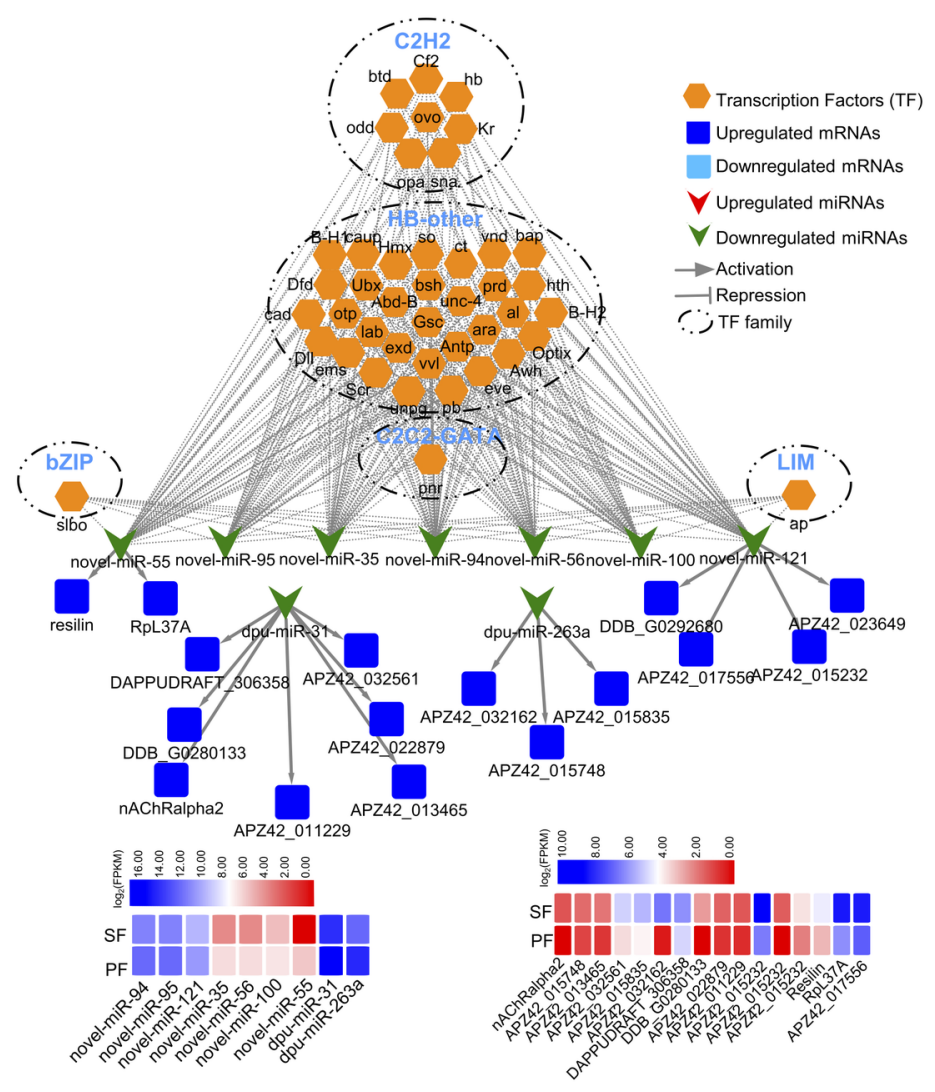

Figure 8. TF-miRNA-mRNA regulatory network in SF vs PF. Gene expression level: $\log _{2}(\mathrm{FPKM})$, when $F P K M<1, \log _{2}(\mathrm{FPKM})=0$.

Figure 5 TF-miRNA-mRNA regulatory network in SF vs PF.

Gene expression level: $\log _{2}(\mathrm{FPKM})$, when $\mathrm{FPKM}<1, \log _{2}(\mathrm{FPKM})=0$. 
Fig.8

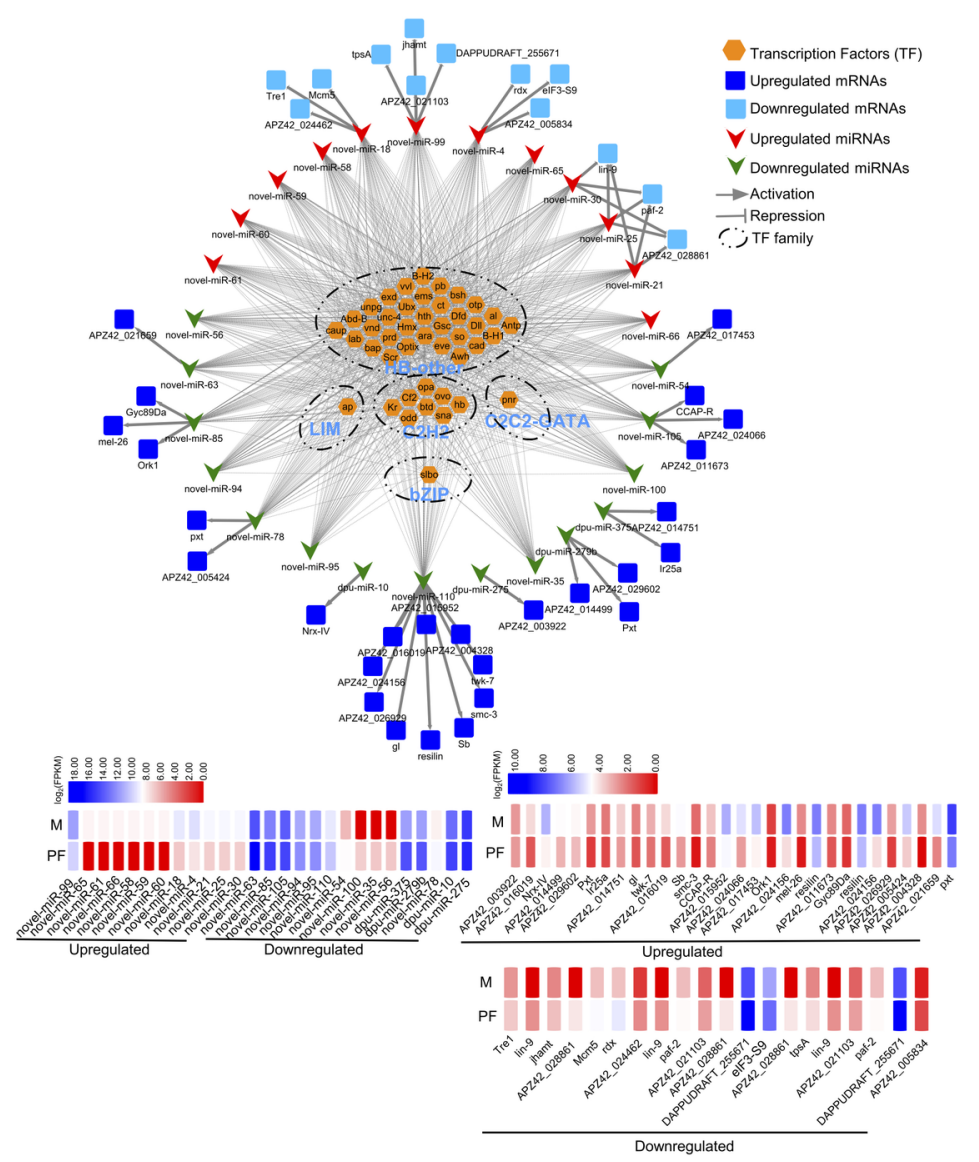

Figure 8. TF-miRNA-mRNA regulatory network in $M$ vs PF. Gene expression level: $\log _{2}(\mathrm{FPKM})$, when $\mathrm{FPKM}<1, \log _{2}(\mathrm{FPKM})=0$.

Figure 6 TF-miRNA-mRNA regulatory network in M vs PF.

Gene expression level: $\log _{2}(\mathrm{FPKM})$, when $\mathrm{FPKM}<1, \log _{2}(\mathrm{FPKM})=0$. 
Fig.9

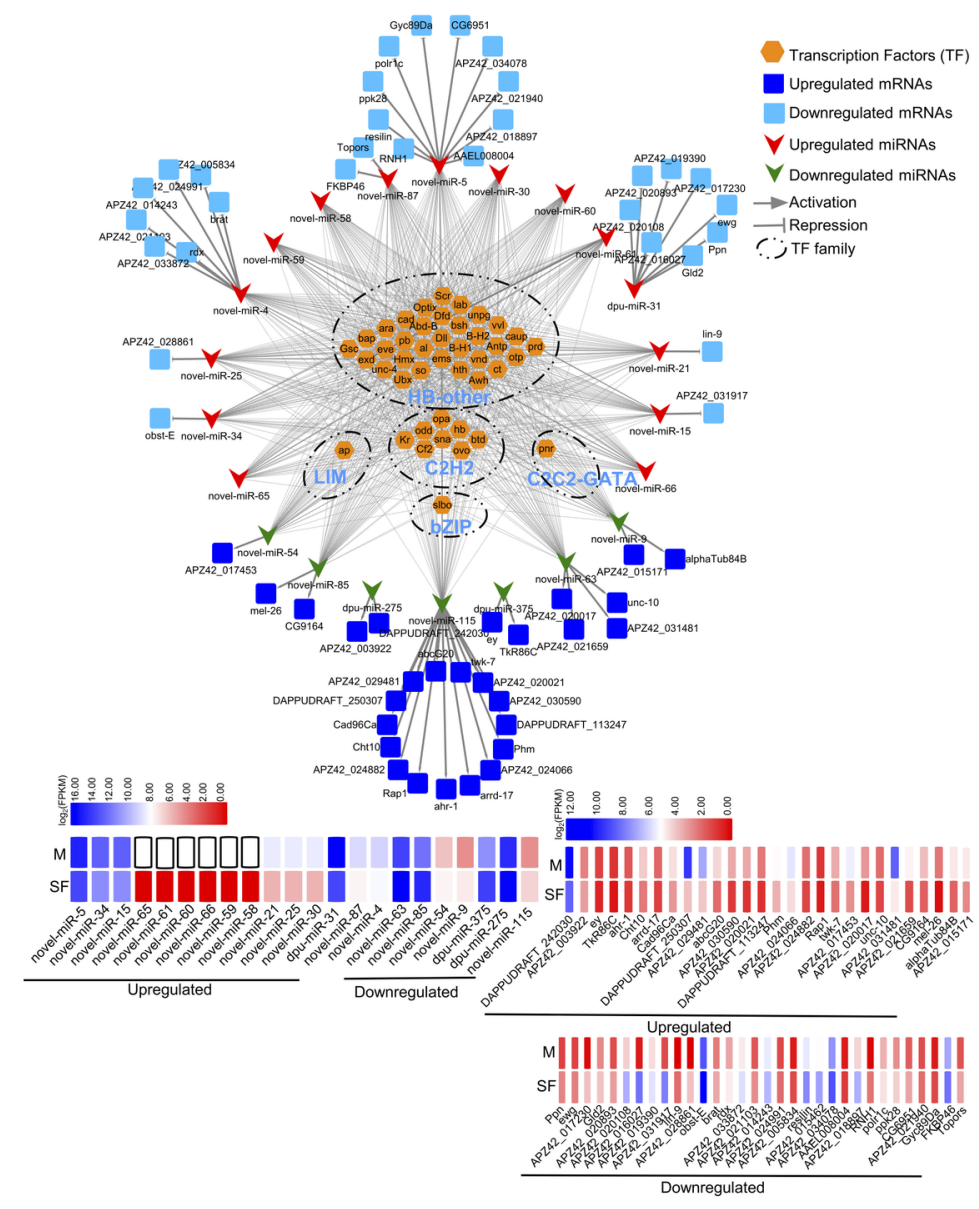

Figure 9. TF-miRNA-mRNA regulatory network in M vs SF. Gene expression level: $\log _{2}(\mathrm{FPKM})$, when FPKM $<1, \log _{2}(\mathrm{FPKM})=0$.

Figure 7 TF-miRNA-mRNA regulatory network in M vs SF.

Gene expression level: $\log _{2}(\mathrm{FPKM})$, when FPKM $<1, \log _{2}(\mathrm{FPKM})=0$. 
(a)

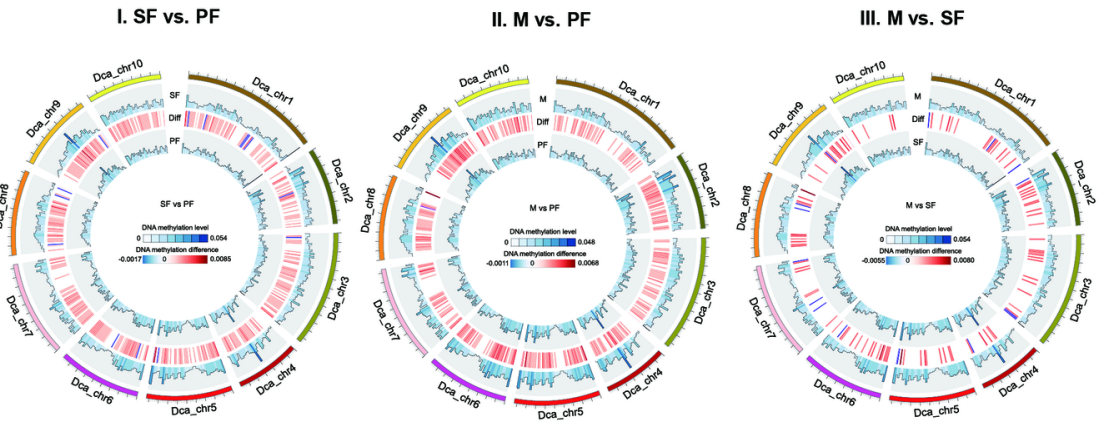

(b)

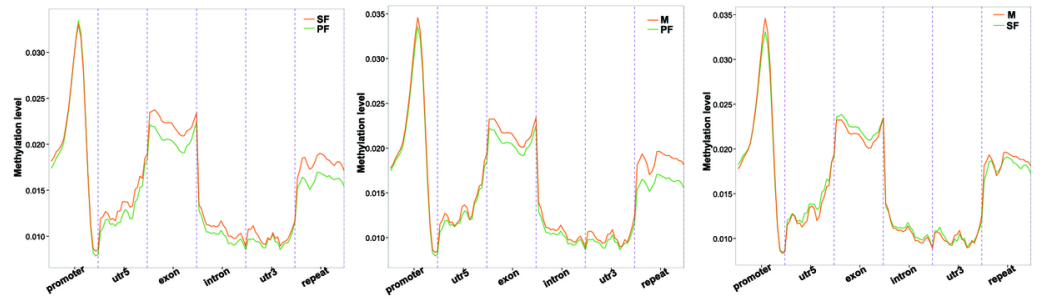

(c)

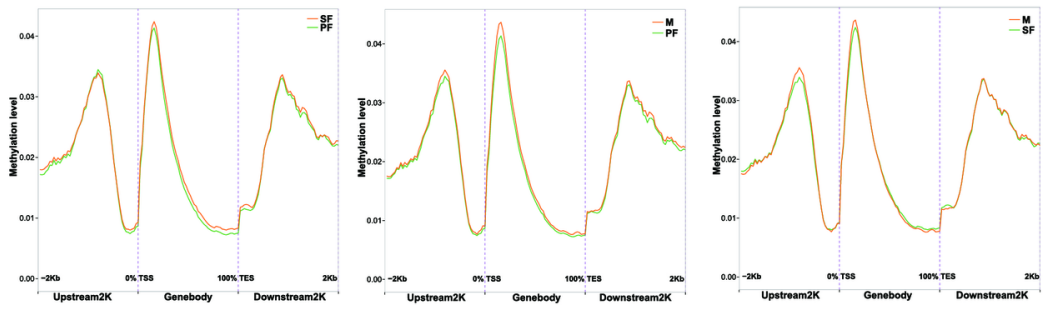

Figure 8 Comparison of DNA methylation.

(a) Circular representation of DNA methylation levels difference for SF vs. PF, M vs. PF and M vs. SF. Methylation features in 200-Kb intervals across the 10 Chromosomes. From outer to inner circles, each $D$. carinata chromosome (Dca_chr1-Dca_chr10), the methylation level in case group, the difference in case and control, the methylation level in contsrol group were exhibited in turn. (b) Distribution of methylation levels on gene functional elements. (c) Distribution of methylation levels around gene bodies. TTS: Transcription termination site. 

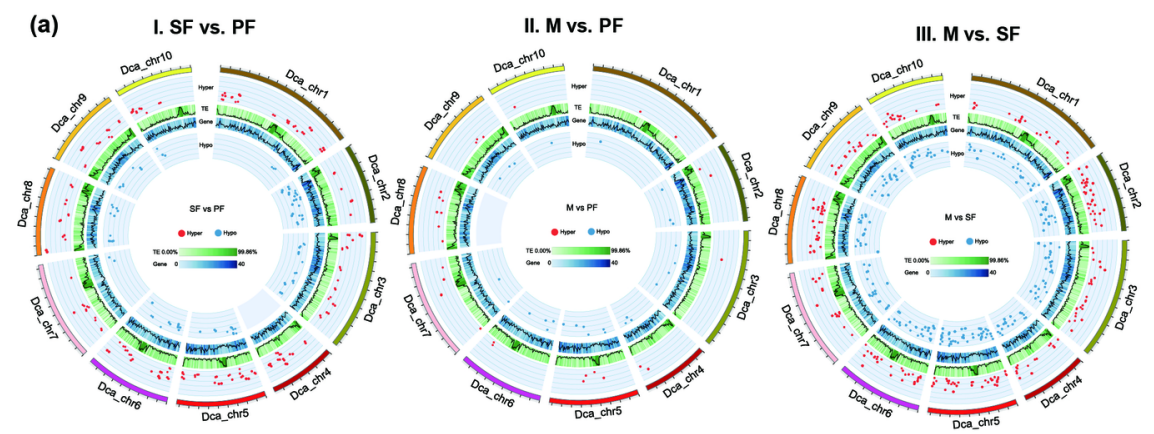

(b)
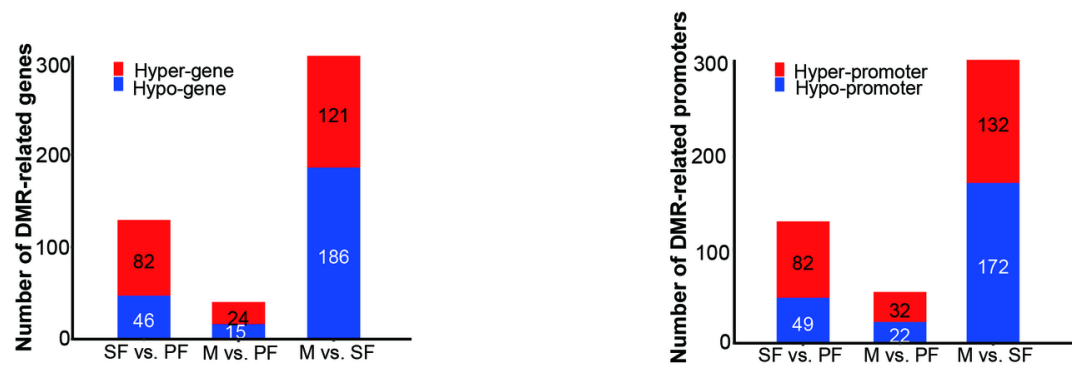

Figure 9 Differential methylation analysis.

(a) The distribution of DNA methylation regions on chromosomes. DMR features in 100-Kb intervals across the 10 Chromosomes. From outer to inner circles, each D. carinata chromosome (Dca_chr1-Dca_chr10), the differential hypermethylationin in case group, Transposon elements (TE) coverage, Gene density and the differential hypomethylation in control group were exhibited in turn. The red dot represent the DMR statistic value $\log 5(\mid$ areaStat $\mid)$, and the higher the outward dot, the more significant the position difference is; the blue dot represent the DMR statistic value log5(|areaStat $\mid)$, and the higher the inward dot, the more significant the position difference is. (b) Number of hyper- and hypo-related genes and promoters.

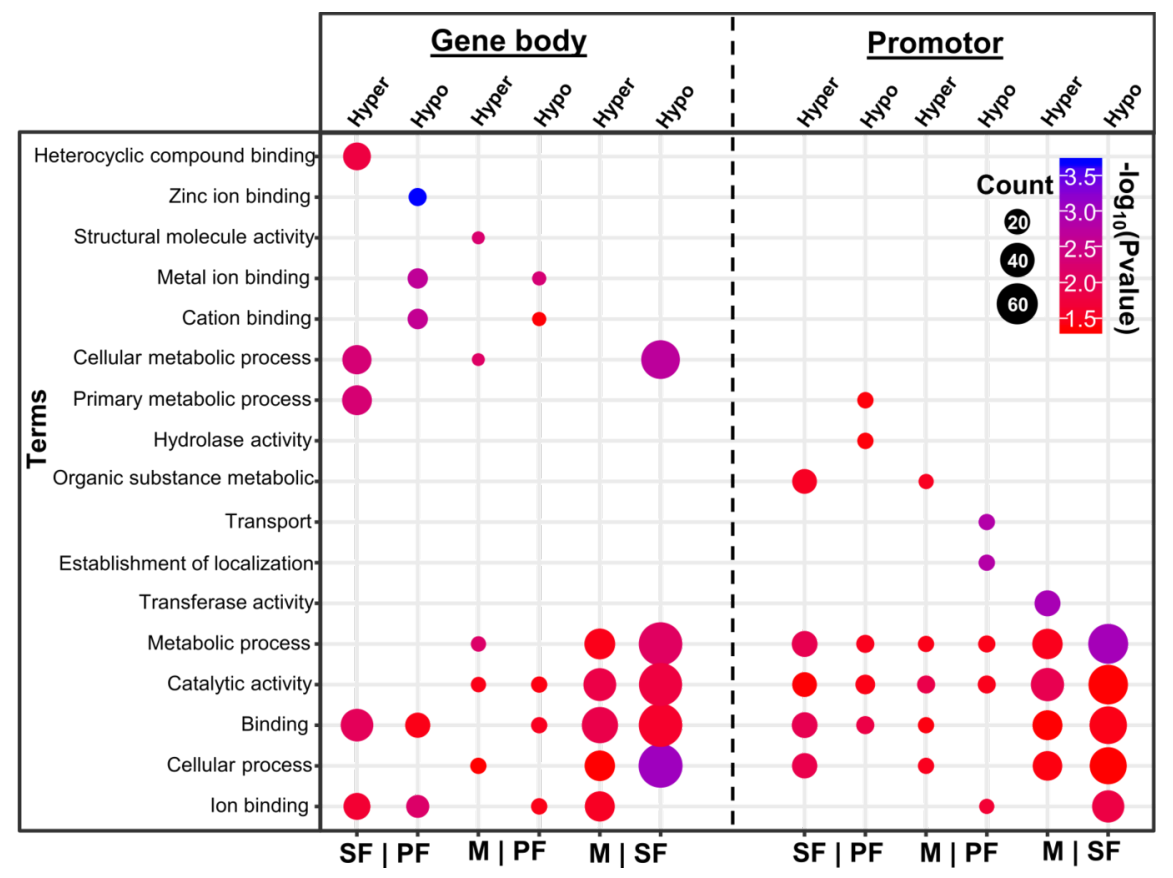


Figure 10 Gene ontology (GO) categories significantly enriched in DMR-associated genes and promoters .

The p-values were calculated using the Benjamini-corrected modified Fisher's exact test.

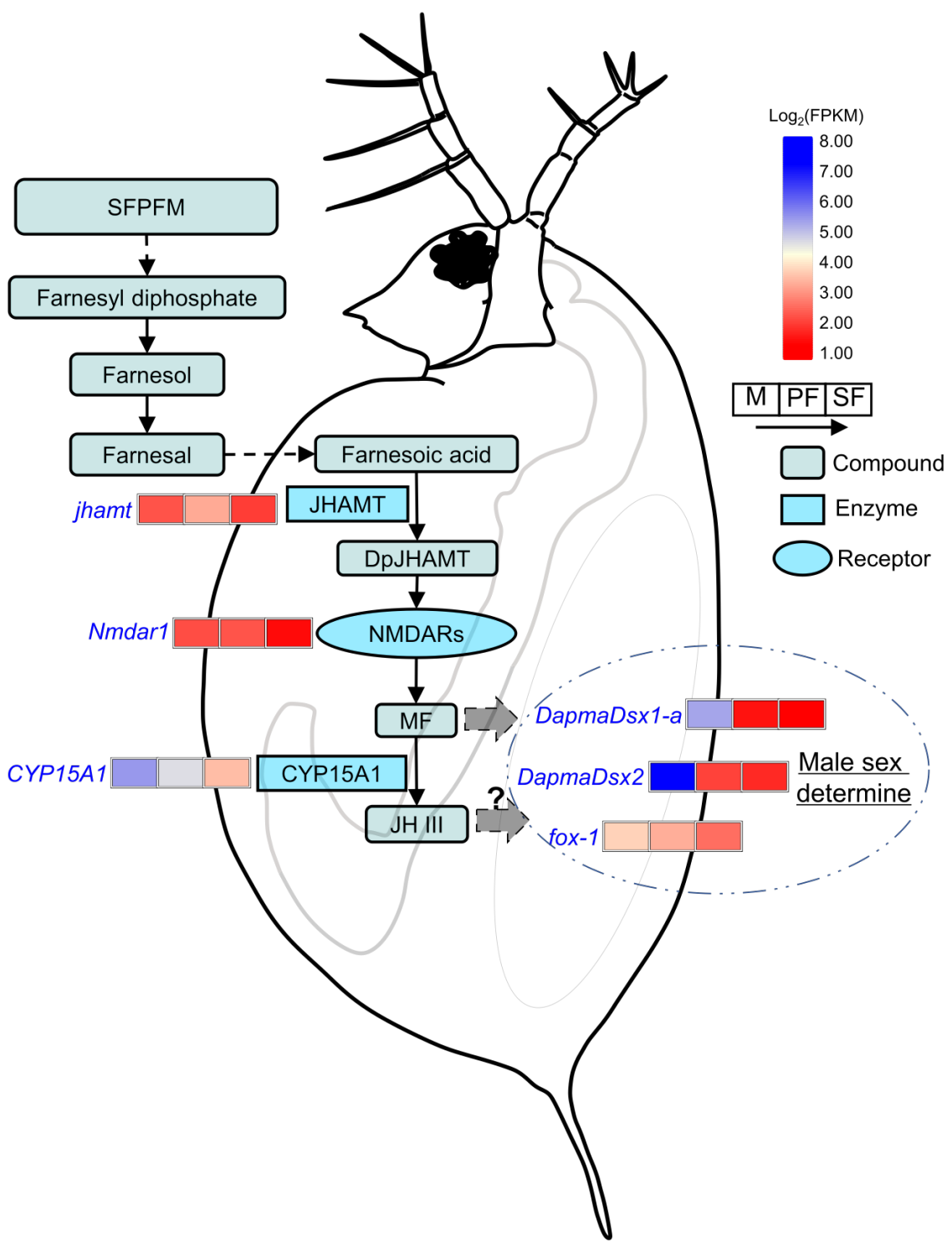

Figure 11 The putative insect hormone synthesis pathway involved in male sex determine process based on the previous and present study.

JHAMT : Juvenile hormone acid O-methyltransferase; CYP15A1 : Methyl farnesoate epoxidase; MF: Methyl farnesoate. Gene expression level: $\log _{2}(\mathrm{FPKM})$, when $\mathrm{FPKM}<1, \log _{2}(\mathrm{FPKM})=0$. 